\title{
AN APPLICATION OF FUNCTIONAL OPERATOR MODELS TO DISSIPATIVE SCATTERING THEORY $\left({ }^{1}\right)$
}

BY

\author{
DANIEL ALAN BONDY
}

\begin{abstract}
An abstract framework for dissipative scattering theory is developed and then applied to two systems previously considered by P. Lax and R. S. Phillips. Results relating the poles and zeroes of the scattering matrix to the spectra of the infinitesimal generators $A$ (which generates the semigroup formed by mapping initial data into solution data at time $t$ ) and $B$ (which generates a "Iocal" semigroup) are proven. In particular these results are shown to follow from the fact that the characteristic function of $A$ (appropriately defined) and the scattering matrix combine to form the characteristic function of $B$.
\end{abstract}

Introduction. In the early 1960's P. D. Lax and R. S. Phillips developed a scattering theory for energy conserving systems in an odd number of space dimensions. (See [6] for details.) In their work major roles are played by two distinguished subspaces $D_{+}$and $D_{-}$. These subspaces are common to both the perturbed system, which operates on the Hilbert space $H$, and the unperturbed system, which operates on the Hilbert space $H_{0}$. Let $U(t)$ be the perturbed unitary group determined by the wave equation in an exterior domain with conservative boundary conditions, and let $U_{0}(t)$ be the unperturbed group determined by the wave equation in free space. Lax and Phillips explicitly find a spectral representation for the unperturbed group $U_{0}(t)$ on $L^{2}(\mathrm{R}, N)$, the Hilbert space of square integrable vector-valued functions on the real line; here $N$ denotes an auxiliary Hilbert space.

The scattering operator $S$ also plays a major role in the Lax-Phillips theory. It is defined as

$$
S h=\lim _{t \rightarrow \infty} U_{0}(-t) J U(2 t) J_{0} U_{0}(-t) h, \quad h \in H_{0},
$$

where $J_{0}$ and $J$ are arbitrary unitary operators from $H_{0}$ to $H$ and $H$ to $H_{0}$ respectively which when restricted to $D_{ \pm}$are the identity. The operator $S$ on $H_{0}$

Received by the editors April 28, 1975.

AMS (MOS) subject classifications (1970). Primary 47A45, 47A40; Secondary 35P25.

Key words and phrases. Characteristic functions, dissipative scattering theory.

(1) This paper constitutes a part of the author's Ph.D. thesis. The author would like to express his thanks to R. S. Phillips and C. Foias for their many helpful conversations. 
commutes with $U_{0}(t)$; it follows that in the spectral representation the corresponding operator, denoted by $S$, acts on $L^{2}(\mathbf{R}, N)$ as multiplication:

$$
(S f)(\sigma)=S(\sigma) f(\sigma), \quad f \in L^{2}(\mathbf{R}, N) .
$$

$S(\sigma)$ is called the scattering matrix. $S(\sigma)$ is shown to have a meromorphic extension to the entire plane which is analytic in the lower half plane. We denote this extension by $S(z)$.

For physical reasons Lax and Phillips studied a semigroup of operators $Z(t)$ defined by

$$
Z(t) h=P_{H \ominus D_{+}}^{H} U(t) P_{H \ominus D_{-}}^{H} h, \quad h \in H ; t \geqslant 0 .
$$

Here $P_{N}^{M}$ denotes the (orthogonal) projection of $M$ onto $N$. Lax and Phillips found that the semigroup $Z(t)$ is related to the scattering matrix $S$ in the following manner:

THEOREM 1. If $B$ is the infinitesimal generator of $Z(t)$, then $z$ belongs to the (point) spectrum of $B$ if and only if -iz is a pole of $S(z)$.

Shortly after the development of the Lax-Phillips theory, a relationship to the theory of unitary dilations was discovered by V. M. Adamjan and D. Z. Arov. (See [9] for details of the theory of unitary dilations.) Before explaining this connection we recall some definitions. Let $Z(t)$ be an arbitrary contraction semigroup defined on a Hilbert space $D$. We say $U(t)$, a unitary group defined on $H \supset D$, is a unitary dilation of $Z(t)$ if

$$
Z(t) d=P_{D}^{H} U(t) d \quad \text { for all } d \in D \text { and } t \geqslant 0 .
$$

To any contraction semigroup $Z(t)$ we associate a contraction operator-namely the Cayley transform of the infinitesimal generator of $Z(t)$. Denote this operator by $Z$ and call $Z$ the cogenerator of $Z(t)$. We define the characteristic function of the contraction $Z$ as

$$
\Theta_{Z}(\lambda)=\left[-Z+\lambda D_{Z *}\left(I-\lambda Z^{*}\right)^{-1} D_{Z}\right]{\left.\right|_{\overline{D_{Z}}}}
$$

where $D_{Z}^{2}=I-Z^{*} Z$. Actually, we define the characteristic function only up to unitary equivalence.

For $\lambda=(z+i) /(z-i)$ we define the characteristic function of $Z(t)$ as

$$
\Theta_{Z(t)}(z)=\Theta_{Z}(\lambda)
$$

We note that $\Theta_{Z}$ is an analytic operator-valued function in the unit disc while $\Theta_{z(t)}$ is analytic in the lower half plane. In [1], [2], and [3], Adamjan and Arov show that for the energy conserving systems in odd space dimensions considered by Lax and Phillips, $U(t)$ is the (minimal) unitary dilation of the semigroup $Z(t)$ and 


$$
\Theta_{\tilde{Z}(t)}^{\tilde{s}}(z)=S(z)
$$

where

$$
\Theta_{Z}^{\tilde{Z}(t)}(x+i y)=\Theta_{Z(t)}(-x+i y)^{*} .
$$

Using this relationship, Theorem 1 becomes a consequence of the Sz.-NagyFoiaş theory of unitary dilations. To see this we recall that Lax and Phillips have shown that $B$ has only point spectrum and $S$ is meromorphic in the entire plane. It is well known from the theory of unitary dilations that $z$ belongs to the point specturm of $B$ if and only if $i z$ is a zero of $\Theta_{Z(t)}$. For the energy conserving Lax-Phillips system, $S$ is unitary on the real axis. By (6) and (7) we see that $\Theta_{\tilde{z}(t)}$ is also unitary on the real axis. Thus we can use the Schwarz reflection principle and conclude that $-i z$ is a pole of $\Theta_{\tilde{Z}(t)}$. By (6) we see that this is the case if and only if $-i z$ is a pole of $S$.

More recently in [8] Lax and Phillips have extended their theory to dissipative systems in odd space dimensions. Again $D_{+}$and $D_{-}$play major roles. Let $T(t)$ be the perturbed group determined by the wave equation in an exterior domain with dissipative boundary conditions and let $U_{0}(t)$ be the unperturbed group. In analogy with the conservative case we define $Z(t)$ and $S$ as follows:

$$
\begin{gathered}
Z(t) h=P_{H \ominus D_{+}}^{H} T(t) P_{H \ominus D_{-}}^{H} h, \quad h \in H ; t \geqslant 0, \\
S h=\lim _{t \rightarrow \infty} U_{0}(-t) J T(2 t) J_{0} U_{0}(-t) h, \quad h \in H_{0} .
\end{gathered}
$$

Simple arguments show that $Z(t)$ can no longer have $S$ as its characteristic function. Nevertheless, Theorem 1 and the following theorem are shown to hold:

THEOREM 2. If $A$ is the infinitesimal generator of $T(t)$ and $z$ belongs to the point spectrum of $A$, then -iz is a zero or possibly a pole of $S$.

This paper explains Theorems 1 and 2 in terms of dilation theory. $\S 1$, due to Foias, puts the Lax-Phillips dissipative theory into an equivalent discrete framework. Let $U(t)$ defined on $K \supset H$ be the (minimal) unitary dilation of $T(t)$. In the discrete framework, instead of considering the semigroups $U(t)$, $T(t)$, and $Z(t)$, we consider their cogenerators $U, T$, and $Z$. The main result of this section shows that $S$ can be characterized as an orthogonal projection onto a subspace of $K$.

In $\$ 2$ the main theorems of the paper are proven. We explicitly construct an operator $\Sigma$ on a Hilbert space $J \subset K$ which has $S$ as its characteristic function. We show $U$ is a unitary dilation of $\Sigma^{*}, Z$, and $T$. These results enable us to generalize (6). If we let $\Theta_{T}$ denote the characteristic function of $T$, the generalization of (6) states (in essence) that 


$$
\Theta_{Z}(\lambda)(U-Z) d=\left(\begin{array}{ll}
S^{\sim}(\lambda) & \Omega_{12}(\lambda) \\
\Omega_{21}(\lambda) & \Theta_{T}(\lambda)
\end{array}\right)\left(\begin{array}{l}
\left(U-\Sigma^{*}\right) d \\
(U-T) d
\end{array}\right)
$$

where $d \in H \Theta\left(D_{-} \oplus D_{+}\right)$and $\Omega_{12}(\lambda)$ and $\Omega_{21}(\lambda)$ are contraction operatorvalued analytic functions in the unit disc. At the end of this section we put (10) into an alternative form:

$$
\Theta_{Z}(\lambda)(U-Z) d=S^{\sim}(\lambda)\left(U-\Sigma^{*}\right) d^{\prime} \oplus \Theta_{T}(\lambda)(U-T) d^{\prime \prime}
$$

where $d=d^{\prime} \oplus d^{\prime \prime}$.

In $\S 3$ we use (11) to find relationships between the poles and zeroes of the characteristic functions of $T, Z$, and the scattering matrix. These relationships are actually discrete versions of Theorems 1 and 2.

In $\$ 4$ we convert the discrete versions of Theorems 1 and 2 into their continuous counterparts. We also show that the abstract framework considered in $\S \S 1-3$ applies to the systems considered in [7] and [8].

1. The discrete framework. In their paper on scattering for dissipative systems (see [8]) Lax and Phillips consider the following abstract framework:

(i) Two strongly continuous groups of unitary operators $U_{ \pm}(t)(t \in R)$ which operate on the Hilbert spaces $H_{ \pm}$and two distinguished subspaces $D_{ \pm} \subset$ $H_{ \pm}$which satisfy

$$
\begin{aligned}
U_{ \pm}(t) D_{ \pm} & \subset D_{ \pm}, \quad t \geqslant 0, \\
\bigcap_{t \in R_{ \pm}} U_{ \pm}(t) D_{ \pm} & =\{0\}, \\
\bigvee_{t \in \mathbb{R}} U_{ \pm}(t) D_{ \pm} & =H_{ \pm} .
\end{aligned}
$$

(ii) A strongly continuous semigroup of contractions $T(t)(t \in \mathbf{R})$ which operates on a Hilbert space $H$ containing $D_{ \pm}$and satisfies

$$
\begin{gathered}
\left.T(t)\right|_{D_{+}}=\left.U_{+}(t)\right|_{D_{+}}, \quad t \in \mathbf{R}_{+}, \\
\left.T^{*}(t)\right|_{D_{-}}=\left.U_{-}(-t)\right|_{D_{-},} \quad t \in \mathbf{R}_{+}, \\
\text {s-lim } P_{H \ominus D_{+}}^{H} T(t)=0, \\
\operatorname{s}_{t \rightarrow \infty} P_{H \ominus \infty}^{H} P_{H}^{*} T^{*}(t)=0,
\end{gathered}
$$

where "s-lim" indicates the limit in the strong operator topology. We call the preceding system a continuous framework. 
As in [3] and [5] we prefer to work in an equivalent discrete framework. This section is largely concerned with showing the equivalence of these two systems. The material is due to $\mathrm{C}$. Foias and is included here only for completeness.

Let $U(t), t \in \mathbf{R}_{+}$, be the minimal unitary dilation of $T(t)$. Then $U(t)$ is, by definition, a strongly continuous group of unitary operators acting on a Hilbert space $K$, which contains $H$ as a subspace, satisfying the following conditions:

$$
\begin{gathered}
T(t)=\left.P_{H}^{K} U(t)\right|_{H}, \quad t \in \mathbf{R}_{+}, \\
K=\bigvee_{t \in \mathbb{R}} U(t) H .
\end{gathered}
$$

By (1.3) and (1.4) we can identify $H_{ \pm}$with $\bigvee_{t \in R} U(t) D_{ \pm}$in such a way that $U_{ \pm}(t)$ coincides with $\left.U(t)\right|_{V_{t \in R} U(t) D}$. Thus we can assume $H_{ \pm} \subset K$ and

$$
\begin{gathered}
H_{ \pm}=\bigvee_{t \in \mathbf{R}} U(t) D_{ \pm}, \\
U_{ \pm}(t)=\left.U(t)\right|_{H \pm}, \quad t \in \mathbf{R} .
\end{gathered}
$$

To find an equivalent discrete framework we need the following

LEMMA 1.1. Let $T(t)$ be a contraction semigroup on $H$ with cogenerator T. Let $D$ be any subspace of $H$. Consider the following statements:

$$
\begin{gathered}
T(t) D \subset D, \quad t \in \mathbf{R}_{+}, \\
\left.T(t)\right|_{D} \text { is an isometry for } t \in \mathbf{R}_{+}, \\
\bigcap_{t \in \mathrm{R}_{+}} T(t) D=\{0\}, \\
\operatorname{s-lim}_{t \rightarrow \infty} P_{H \Theta D}^{H} T(t)=0, \\
T D \subset D, \\
\bigcap_{n \in \mathbf{Z}_{+}} T^{n} D=\{0\} ; \quad Z_{+}=\{n ; n \geqslant 0\}, \\
s-\lim P_{H \in D^{H}}^{H} T^{n}=0 .
\end{gathered}
$$

Then the following relationships hold: 


$$
\begin{aligned}
(1.10) & \Longleftrightarrow(1.14), \\
(1.10) \&(1.11) & \Longleftrightarrow(1.14) \&(1.15), \\
(1.10) \&(1.11) \&(1.12) & \Longleftrightarrow(1.14) \&(1.15) \&(1.16),
\end{aligned}
$$$$
\text { (1.10) \& (1.11) \& (1.12) \& (1.13) } \Longleftrightarrow(1.14) \&(1.15) \&(1.16) \&(1.17) \text {. }
$$

Proof. See [5].

For later reference we note that if we set

$$
\begin{aligned}
& e_{t}(\lambda)=\exp \left(t \frac{\lambda+1}{\lambda-1}\right), \quad|\lambda|<1, t \in \mathbf{R}_{+}, \\
& \phi_{s}(\lambda)=(\lambda-1+s)(\lambda-1-s)^{-1}, \quad|\lambda|<1 ; s \in \mathbf{R}_{+},
\end{aligned}
$$

then by Theorem III.8.1 of [9] we conclude

$$
\begin{aligned}
T(t) & =e_{t}(T)=\underset{\gamma \rightarrow 1^{-}}{s-\lim _{j}} e(\gamma T), \quad t \in \mathbf{R}_{+} . \\
T & =\underset{s-\lim _{s}}{ } \phi_{s}(T(s)) .
\end{aligned}
$$

Let $U$ be the cogenerator of $U(t)$ and $T$ be the cogenerator of $T(t)$. Then by $\S I I I .9 .1$ of [9] we can conclude that $U$ on $K$ is the minimal unitary dilation of $T$. Thus, by definition, we have

$$
\begin{gathered}
T^{n}=\left.P_{H}^{K} U^{n}\right|_{H}, \quad n \in \mathbf{Z}_{+}, \\
K=\bigvee_{n \in \mathbf{Z}} U^{n} H .
\end{gathered}
$$

Lemma 1.2. Let $D \subset H$ be a subspace enjoying property (1.10). Then

$$
\bigvee_{t \in \mathbb{R}} U(t) D=\bigvee_{n \in \mathbf{Z}} U^{n} D
$$

Proof. See [5].

From the two preceding lemmas it is clear that by considering the cogenerators $U$ and $T$ of $U(t)$ and $T(t)$ respectively we arrive at the following completely equivalent discrete framework.

There is a contraction $T$ on a Hilbert space $H$ and two distinguished subspaces $D_{ \pm} \subset H$ such that

$$
\begin{gathered}
T D_{+} \subset D_{+} ; \quad T^{*} D_{-} \subset D_{-} \\
\left.T\right|_{D_{+}} \text {and }\left.T^{*}\right|_{D_{-}} \text {are isometries, } \\
\bigcap_{n \in \mathrm{Z}_{+}} T^{n} D_{+}=\{0\}=\bigcap_{n \in \mathrm{Z}_{+}} T^{* n} D_{-},
\end{gathered}
$$




$$
\operatorname{s-lim}_{n \rightarrow \infty} P_{H \Theta D_{+}}^{H} T^{n}=0=\operatorname{slim}_{n \rightarrow \infty} P_{H \ominus D_{-}}^{H} T^{* n} .
$$

In [8], under the assumptions of the continuous abstract framework, the following wave operators are shown to exist.

$$
\begin{aligned}
& W_{-}=\operatorname{s-lim}_{t \rightarrow \infty} U_{-}(t) P_{D_{-}}^{H} T^{*}(t), \\
& W_{+}=\text {s-lim } U_{t \rightarrow \infty}(-t) P_{D_{+}}^{H} T(t) .
\end{aligned}
$$

By (1.8) and (1.9) in conjunction with (1.5) we arrive at

$$
\begin{aligned}
& W_{-}=s-\lim _{t \rightarrow \infty} U(t) T^{*}(t), \\
& W_{+}=s-\lim U(-t) T(t) .
\end{aligned}
$$

Now by Proposition III.9.1 of [9] we conclude

$$
\begin{aligned}
& W_{-}=s-\lim U_{n \rightarrow \infty}^{n} T^{* n}, \\
& W_{+}=s-\lim _{n \rightarrow \infty} U^{* n} T^{n} .
\end{aligned}
$$

We note that $W_{-}$is a contraction operator from $H$ to $H_{-}$, and $W_{+}$is a contraction operator from $H$ to $H_{+}$. We define the scattering operator $S$ as

$$
S=W_{+} W_{-}^{*}
$$

and note that $S$ is a contraction operator from $H_{-}$to $H_{+}$.

The remainder of this section is devoted to a closer study of $S$. If $T$ is a contraction operator on $H$, and $U$, operating on $K$, is its minimal unitary dilation, the structure of $K$ is well known. (See Chapter II of [9].) In particular it is known that

$$
L=\overline{(U-T) H} \text { and } \quad L_{*}=\overline{\left(I-U T^{*}\right) H}
$$

are wandering subspaces for $U$ i.e., $U^{n} L \perp L$ for all $n \in Z \backslash\{0\}$ and similarly for $L_{*}$. If we let

$$
K_{+}=\bigvee_{n \in Z_{+}} U^{n} H
$$

it is well known that

$$
K_{+}=M_{+}\left(L_{*}\right) \oplus R=H \oplus M_{+}(L),
$$

where

and for any subspace $P$

$$
R=\bigcap_{n \in \mathbf{Z}_{+}} U^{n} K_{+}
$$




$$
M_{+}(P)=\bigvee_{n \in \mathrm{Z}_{+}} U^{n} P
$$

We note that $M_{+}(L)=\bigoplus_{0}^{\infty} U^{n} L$ since $L$ is wandering. Furthermore if we define

$$
U_{+}=U_{K_{+}}
$$

then $U_{+}$is the minimal isometric dilation of $T$; i.e. $U_{+}$satisfies

$$
\begin{gathered}
T^{n}=\left.P_{H}^{K_{+}} U_{+}^{n}\right|_{H}, \quad n \in \mathbf{Z}_{+}, \\
K_{+}=\bigvee_{n \in \mathbf{Z}_{+}} U^{n} H
\end{gathered}
$$

The following relations also hold:

$$
P_{H}^{K^{+}} U_{+}=T P_{H}^{K_{+}} ; \quad T^{*}=\left.U_{+}^{*}\right|_{H}
$$

where we write $U_{+}^{*}$ for $\left(U_{+}\right)^{*}$. We also have

$$
\left.P_{R}^{K}{ }^{+}\right|_{H}=\operatorname{s-lim}_{n \rightarrow \infty} U^{n} T^{* n}
$$

For a proof of (1.37) and (1.38) see Chapters I.4 and II.3 respectively of [9].

LEMMA 1.3. Let $D_{*}$ be a subspace of $H$ which satisfies (1.14), (1.15), and (1.16) with respect to $T^{*}$ (instead of $T$ ). Then $D_{*}$ satisfies (1.17) with respect to $T^{*}$ if and only if

$$
R=H_{*} \stackrel{\text { def }}{=} \bigvee_{n \in \mathrm{Z}} U^{n} D_{*}
$$

Proof. See [5].

Let $R_{*}$ denote the subspace of $K$ corresponding to $R$ in case we replace $T$ by $T^{*}$ and $U$ by $U^{*}$. Then we have

$$
K=R \oplus M\left(L_{*}\right)=R_{*} \oplus M(L)=M_{-}\left(L^{*}\right) \oplus H \oplus M_{+}(L)
$$

where $M_{ \pm}(P) \stackrel{\text { def }}{=} \vee n \in \mathrm{z}_{ \pm} U^{n} P$ for any subspace $P, M(P) \stackrel{\text { def }}{=} M_{+}(P) \vee M_{-}(P)$, and $L^{*}=U^{*} L_{*}$. We note that $M(L)=\bigoplus_{-\infty}^{\infty} U^{n} L$ since $L$ is a wandering subspace.

We now state the main theorem of this section.

THEOREM 1.4. The wave operators $W_{ \pm}$defined by (1.28) are given by

$$
W_{+}=P_{R_{*}}^{K} I_{H}, \quad W_{-}=\left.P_{R}^{K}\right|_{H}
$$

Furthermore, the scattering operator $S$ defined by (1.29) is given by

$$
S=\left.P_{R}^{K}\right|_{R} \text {. }
$$


Proof. See [5].

2. A decomposition for $\theta_{z}$. We begin with a quick review of the basic definitions and theorems from the theory of characteristic functions and functional models. See Chapter VI of [9] for complete details.

Let $T$ be an arbitrary contraction operator defined on a Hilbert space $H$ and define

$$
\begin{aligned}
D_{T}=\left(I-T^{*} T\right)^{1 / 2}, & D_{T^{*}}=\left(I-T T^{*}\right)^{1 / 2}, \\
D_{T}=\overline{D_{T} H}, & D_{T^{*}}=\overline{D_{T^{*} H}} .
\end{aligned}
$$

We call the contraction operator-valued function

$$
\Theta_{T}(\lambda)=\left[-T+\lambda D_{T^{*}}\left(I-\lambda T^{*}\right)^{-1} D_{T}\right]_{D_{T}} \quad|\lambda|<1,
$$

the characteristic function of $T$. We use the notation $\left\{D_{T}, D_{T^{*}}, \Theta_{T}(\lambda)\right\}$ to denote the fact that $\Theta_{T}(\lambda)$ maps from $D_{T^{*}}$. We say that the operator-valued analytic functions $\left\{D_{T}, D_{T^{*}}, \Theta_{T}(\lambda)\right\}$ and $\left\{U, U_{*}, \Omega(\lambda)\right\}(|\lambda|<1)$ coincide if there exists a unitary mapping $V$ from $D_{T}$ to $U$ and a unitary mapping $V_{*}$ from $D_{T^{*}}$ such that

$$
\Omega(\lambda)=V_{*} \Theta_{T}(\lambda) V^{-1}, \quad|\lambda|<1 .
$$

As in $\S 1$, we let $U$ on $K$ be the minimal unitary dilation of $T$ on $H$. It is clear that we can define unitary mappings $V$ and $V_{*}$ as follows:

$$
\begin{gathered}
V: D_{T} \rightarrow \mathrm{L} \stackrel{\text { def }}{=} \overline{(U-T) H,} \\
D_{T} h \mapsto(U-T) h
\end{gathered}
$$

and

$$
\begin{aligned}
V_{*}: D_{T^{*}} & \rightarrow L_{*} \stackrel{\text { def }}{=} \overline{\left(U^{*}-T^{*}\right) H}, \\
D_{T^{*}} h & \mapsto\left(I-U T^{*}\right) h
\end{aligned}
$$

so that, $\left\{L, L_{*}, \Omega(\lambda)\right\}$ defined by (2.4) coincides with $\Theta_{T}(\lambda)$. To simplify notation we will denote $\Theta_{T}(\lambda)$ and any other operator-valued analytic function $\Omega(\lambda)$ which coincides with $\Theta_{T}(\lambda)$ by the same symbol, namely $\Theta_{T}(\lambda)$. Thus we define $\Theta_{T}(\lambda)$ only up to unitary equivalence.

For any analytic operator-valued function $\Theta(\lambda)$ we define

$$
\Theta^{\sim}(\lambda)=\Theta(\bar{\lambda})^{*}, \quad|\lambda|<1 .
$$

It is well known that

$$
\Theta_{T^{*}}(\lambda)=\Theta_{T}^{\tilde{T}}(\lambda), \quad|\lambda|<1
$$


With $\left\{D_{T}, D_{T^{*}}, \Theta_{T}(\lambda)\right\}$ we associate the operator $\Theta_{T}$ from $L^{2}\left(D_{T}\right)(=$ square inte-

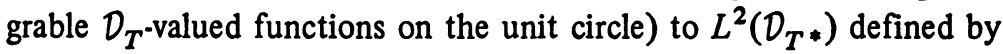

$$
\left(\Theta_{T} u\right)\left(e^{i t}\right)=\Theta_{T}\left(e^{i t}\right) u\left(e^{i t}\right), \quad u \in L^{2}\left(D_{T}\right) .
$$

Notice that the strong radial limits of $\Theta_{T}(\lambda)$ exist almost everywhere on the unit circle so that the right side of (2.9) is well defined.

Let $V$ be a bilateral shift on a Hilbert space $K$. Then $K=M(N)$ for some subspace $N$ of $K$. We define a canonical unitary map $\Phi^{N}$ from $K(=M(N))$ to $L^{2}(N)$ by

$$
\left[\Phi^{N} \sum_{-\infty}^{\infty} V^{k} a_{k}\right](t)=\sum_{-\infty}^{\infty} e^{i k t} a_{k}
$$

where, of course, $a_{k} \in N$ and $\Sigma\left\|a_{k}\right\|^{2}<\infty$. We note that $\Phi^{N}$ turns $V$ on $K$ into multiplication by $e^{i t}$ on $L^{2}(N)$.

We now recall

LEMma 2.1. Let $V_{1}$ and $V_{2}$ be bilateral shifts on the (complex, separable) Hilbert spaces $K_{1}$ and $K_{2}$ with wandering subspaces $N_{1}$ and $N_{2}$ respectively. Let $Q$ be a contraction mapping $K_{1}$ into $K_{2}$ such that

$$
\begin{gathered}
Q V_{1}=V_{2} Q \\
Q M_{+}\left(N_{1}\right) \subset M_{+}\left(N_{2}\right) .
\end{gathered}
$$

Then there exists a contractive operator-valued analytic function $\left\{N_{1}, N_{2}, \Theta(\lambda)\right\}$ in the unit disc such that

$$
\Phi^{N_{1}} Q=\Theta \Phi^{N_{2}}
$$

We call $\left\{N_{1}, N_{2}, \Theta(\lambda)\right\}$ the Fourier representation of $Q$ with respect to $N_{1}$ and $N_{2}$.

CoROllaRY 2.2. Under the assumptions of Lemma 2.1, let $\left\{N_{1}, N_{2}\right.$, $\Theta(\lambda)\}$ be the Fourier representation of $Q$ with respect to $N_{1}$ and $N_{2}$. Let $\left\{V_{1}^{k} N_{1}\right.$, $V_{2}^{* k} N_{2}, \Omega(\lambda)$ \} be the Fourier representation of $Q$ with respect to $V_{1}^{k} N_{1}$ and $V_{2}^{* k} N_{2}$ for $k \in Z_{+}$. Then the following relationship holds:

$$
\lambda^{2 k} \Omega(\lambda)=\Theta(\lambda), \quad|\lambda|<1
$$

We recall that if $T$ is an arbitrary contraction operator on $H$, it has a minimal unitary dilation $U$ defined on $K$. Defining $L$ and $L_{*}$ as in (1.30), we recall the following

Proposition 2.3. If $Q=\left.P_{M\left(L_{*}\right)}\right|_{M(L)}$, then the Fourier representation of 
$Q$ with respect to $L_{*}$ and $L$ is given by (2.4)-(2.6) and therefore is (coincides with) the characteristic function of $T$ given by (2.3).

Since $\Theta_{T}(\lambda)$ is analytic in the unit disc it has a power series representation

$$
\Theta_{T}(\lambda)=\sum_{n=0} \lambda^{n} \Theta_{n}
$$

It is well known that

$$
\Theta_{n}=\left.P_{L_{*}} U^{* n}\right|_{L}, \quad n=0,1,2, \ldots,
$$

where for any $h \in H$

$$
\Theta_{n}(U-T) h= \begin{cases}-\left(I-U T^{*}\right) T h, & n=0, \\ \left(I-U T^{*}\right) T^{* n-1} D_{T}^{2} h, & n=1,2, \ldots\end{cases}
$$

Finally we shall need

Proposition 2.4. Let $T$ be a completely nonunitary contraction on $H$. Denote by $\Gamma_{T}$ the set of points $\mu$ belonging to the open unit disc for which the operator $\Theta_{T}(\mu)$ is not boundedly invertible, together with those $\mu$ on the unit circle (denoted by $C$ ) not lying on any open arcs of $C$ on which $\Theta_{T}(\lambda)$ is a unitary operator-valued analytic function of $\lambda$ Furthermore, denote by $\Gamma_{T}^{0}$, the set of points $\mu$ belonging to the open unit disc for which $\Theta_{T}(\mu)$ is not invertible at all. Then

$$
\sigma(T)=\Gamma_{T} \text { and } \sigma_{p}(T)=\Gamma_{T}^{0}
$$

This concludes the review of the basic theorems from the theory of characteristic functions and functional models.

We now apply these results to the discrete Lax-Phillips framework considered in $\$ 1$. In their paper on scattering for dissipative systems (see [9]) Lax and Phillips prove Theorems 1 and 2 of the Introduction for odd space dimensions only. This restriction is equivalent to assuming

$$
D_{+} \perp D_{-} \text {. }
$$

Unfortunately, for potential scattering in odd space dimensions (see [7] and Chapter VI.2 of [6]) (2.18) no longer holds. In an attempt to make this abstract framework applicable to potential scattering problems we do not assume (2.18). Nevertheless, one cannot expect to obtain analytic functions without some kind of orthogonality assumptions. So instead of (2.18) we make the following (weaker) assumption:

There exist subspaces $E_{ \pm}$with the following properties:

$$
E_{ \pm} \subset D_{ \pm}
$$


(2.20) cts

$$
\begin{aligned}
T(t) E_{+} \subset E_{+} ; & T^{*}(t) E_{-} \subset E_{-}, \quad t>0, \\
& E_{+} \perp E_{-} .
\end{aligned}
$$

We note that Lemma 1.1 allows us to rewrite $(2.20)_{\text {cts }}$ as

$$
T E_{+} \subset E_{+} ; \quad T^{*} E_{-} \subset E_{-} .
$$

We note that $E_{ \pm}$satisfying the analogue of (1.16). To see this we note that

$$
\begin{aligned}
& \{0\}=\bigcap_{n \in \mathbf{Z}_{+}} T^{n} D_{+} \supset \bigcap_{n \in \mathbf{Z}_{+}} T^{n} E_{+} \supset\{0\}, \\
& \{0\}=\bigcap_{n \in \mathbf{Z}_{+}} T^{* n} D_{-} \supset \bigcap_{n \in \mathbf{Z}_{+}} T^{* n} E_{-} \supset\{0\}
\end{aligned}
$$

by (1.16) applied to $D_{ \pm}$and (2.19). Since by (1.15) $T$ (resp. $T^{*}$ ) is an isometry on $D_{+}$(resp. $D_{-}$) we see the above relations imply that $T$ (resp. $T^{*}$ ) is a unilateral shift on $D_{+}$and $E_{+}$(resp. $D_{-}$and $E_{-}$). We will introduce the associated wandering subspaces a little later.

We explicitly do not assume that $E_{ \pm}$satisfy the analogue of (1.17).

For the systems considered in [8], (2.18) holds and we can set

$$
E_{ \pm}=D_{ \pm} \text {. }
$$

For such a choice of $E_{ \pm}$, it is clear that (2.19)-(2.20) hold.

For the case of potential scattering considered in [7] $D_{ \pm}$correspond to what Lax and Phillips denote by $D_{ \pm}^{\prime}$ while $E_{ \pm}$correspond to $D^{\prime \prime}$. That (2.19)(2.20) are satisfied is proven in $\$ 3$ of [7].

The reader is urged to keep these two examples in mind while we proceed in a more general context.

We introduce the following notation:

$$
\begin{array}{ll}
O_{ \pm}(k)=U^{ \pm k} E_{ \pm} \ominus U^{ \pm(k+1)} E_{ \pm}, & k \in \mathbf{Z}_{+}, \\
E_{ \pm}(k)=U^{ \pm k} E_{ \pm}\left(=\bigoplus_{j=k}^{\infty} O_{ \pm}(j)\right), & k \in \mathbf{Z}_{+}, \\
E_{0}(k)=H \ominus\left[E_{+}(k) \oplus E_{-}(k)\right], & k \in \mathbf{Z}_{+}, \\
N_{ \pm}(k)=U^{ \pm k} D_{ \pm} \ominus U^{ \pm(k+1)} D_{ \pm}, & k \in \mathbf{Z}_{+}, \\
D_{ \pm}(k)=U^{ \pm k} D_{ \pm}\left(=\bigoplus_{j=k}^{\infty} N_{ \pm}(j)\right), & k \in \mathbf{Z}_{+} .
\end{array}
$$

We note that $O_{ \pm}(k)$ and $N_{ \pm}(k)$ are wandering subspaces for $U$, the unitary dilation of $T$.

By using (1.39) and (2.26) we can rewrite (1.45) as

$$
S=\left.P_{M\left(N_{+}\right)}^{K}\right|_{M\left(N_{-}\right)} \text {. }
$$


We suppress the suffix $k$ since $M\left(N_{-}(k)\right)=M\left(N_{-}(j)\right)$ for any $j, k \in \mathbf{Z}_{+}$.

At this point we would like to use Lemma 2.1 to obtain the Fourier representation of $S$ with respect to $N_{\ddagger}$. Unfortunately condition (2.12) is not in general satisfied. We note that for the case of odd space dimensions considered by Lax and Phillips in [8], (2.18) holds and this implies (2.12). In general we can not expect the Fourier representation to be analytic.

Nevertheless we can continue to deal with analytic functions by defining

$$
\alpha=\left.P_{M\left(N_{-}\right)}^{K}\right|_{M\left(0_{-}\right)}, \quad \beta=\left.P_{M\left(0_{+}\right)}^{K}\right|_{M\left(N_{+}\right)} .
$$

If we set

$$
S_{\text {red }}=\beta S \alpha
$$

then it is easy to see that

$$
S_{\mathrm{red}}=\left.P_{M\left(O_{+}\right)}^{K}\right|_{M\left(O_{+}\right)}
$$

Taking adjoints we see

$$
S_{\text {red }}^{*}=\left.P_{M\left(O_{-}\right)}^{K}\right|_{M\left(O_{-}\right)} .
$$

ClaIM 2.5. $\alpha, \beta$, and $S_{\text {red }}$ have analytic Fourier representations which we denote as follows:

$$
\begin{gathered}
\left\{O_{-}(k), O_{+}(k-1), S_{\text {red }}^{(k)}(\lambda)\right\}, \quad k=1,2, \ldots, \\
\left\{N_{+}(k-1), O_{+}(k-1), \beta^{(k)}(\lambda)\right\}, \quad k=1,2, \ldots, \\
\left\{O_{-}(k), N_{-}(k), \alpha^{(k)}(\lambda)\right\}, \quad k=1,2, \ldots .
\end{gathered}
$$

Further $\alpha\left(e^{i t}\right)$ and $\beta^{*}\left(e^{i t}\right)$ are isometric almost everywhere on the unit circle.

Proof. To prove that $\alpha, \beta$, and $S_{\text {red }}$ have analytic Fourier representations we apply Lemma 2.1. First we prove (2.33) and (2.34). With the notation as in Lemma 2.1 let $Q=\alpha$ (resp. $\beta$ ) given by (2.29) and let $K_{1}=M\left(O_{-}\right)$(resp. $M\left(N_{+}\right)$) and $K_{2}=M\left(N_{-}\right)$(resp. $M\left(O_{+}\right)$). Let $V_{1}=V_{2}=U$, the minimal unitary dilation of $T$. It is clear that $U$ acts as a bilateral shift on $M\left(O_{ \pm}\right)$and $M\left(N_{ \pm}\right)$. It is also clear that these subspaces reduce $U$. Since $Q$ (i.e. $\alpha$ (resp. $\left.\beta\right)$ ) is a projection we see that $(2.11)$ is satisfied. Thus to apply Lemma 2.1 we need only show that (2.12) is satisfied.

To this end we note that

$$
D_{+}(k-1)^{\perp} \perp E_{+}(k-1) ; \quad E_{-}(k) \perp D_{-}(k)^{\perp}
$$

for $k=1,2, \ldots$ We recall $(2.23),(2.24),(2.26),(2.27)$ and rewrite the previous relationships as

$$
\bar{M}_{-}\left(N_{+}(k-1)\right) \perp M_{+}\left(O_{+}(k-1)\right) ; \quad M_{-}\left(O_{-}(k)\right) \perp \bar{M}_{+}\left(N_{-}(k)\right)
$$

where we define $\bar{M}_{ \pm}(P)=\bigoplus_{k= \pm 1}^{ \pm \infty} U^{k} P$ for any unitary operator $U$ and any wandering subspace $P$. From (2.35) and (2.29) we see 


$$
\beta\left(M_{+}\left(N_{+}(k-1)\right)\right) \subset M_{+}\left(O_{+}(k-1)\right) ; \quad \alpha\left(M_{+}\left(O_{-}(k)\right)\right) \subset M_{+}\left(N_{-}(k)\right) .
$$

for $k=1,2, \ldots$. Thus we see that (2.12) is satisfied and we can conclude that $\alpha$ and $\beta$ have analytic Fourier representations.

To prove the last statement of the claim we note that by $(2.19) M\left(O_{+}\right) \subset$ $M\left(N_{+}\right) ; M\left(O_{-}\right) \subset M\left(N_{-}\right)$and so $\beta^{*}$ and $\alpha$ are isometries. Thus their Fourier representations, considered as mappings from $L^{2}\left(O_{+}(k-1)\right)$ to $L^{2}\left(N_{+}(k-1)\right)$ and $L^{2}\left(O_{-}(k)\right)$ to $L^{2}\left(N_{-}(k)\right)$ respectively, must also be isometries. By a simple localization argument we see that $\alpha\left(e^{i t}\right)$ and $\beta\left(e^{i t}\right)$ are isometric almost everywhere on the unit circle.

We now proceed to the proof of (2.32). Again we use the notation of Lemma 2.1. Let $Q=S_{\text {red }}$ which is defined by (2.31). Let $K_{1}=M\left(O_{-}\right), K_{2}$ $=M\left(O_{+}\right)$. Finally let $V_{1}=V_{2}=U^{*}$. Clearly $U^{*}$ acts a bilateral shift on the $U^{*}$-reducing subspaces $M\left(O_{ \pm}\right)$. Since $Q$ (i.e. $S_{\text {red }}$ ) is a projection involving these subspaces we conclude that (2.11) holds. We now show that (2.12) holds also. We recall that the action of $U$ and $T$ coincide on $D_{+}$while the action of $U^{*}$ and $T^{*}$ coincide on $D_{-}$. Since $E_{ \pm} \subset D_{ \pm}$we can rewrite (2.20) as

$$
U E_{+} \subset E_{+}, \quad U^{*} E_{-} \subset E_{-} .
$$

Thus it is clear from (2.24) that

$$
E_{+}(k-1) \perp E_{-}(k), \quad k=1,2, \ldots
$$

Now (2.38) can be rewritten as

$$
M_{-}\left(O_{-}(k)\right) \perp M_{+}\left(O_{+}(k-1)\right) .
$$

Recalling the definition of $S_{\text {red }}$ we see that this implies

$$
S_{\text {red }}\left(M_{-}\left(O_{-}(k)\right)\right) \subset M_{-}\left(O_{+}(k-1)\right)
$$

which is precisely (2.11) with the appropriate substitutions. This concludes the proof of (2.32) and Claim 2.5.

We note that the Fourier representations of Claim 2.5 depend upon $k=1$, $2, \ldots$ By Corollary 2.2 we see that the different $S_{\text {red }}^{(k)}(\lambda)$ vary only by factors of $\lambda$. Similar statements apply to $\alpha^{(k)}(\lambda)$ and $\beta^{(k)}(\lambda)$.

Having introduced $S_{\text {red }}$, we have no further need of $D_{ \pm}$in this section and will deal with $E_{ \pm}$from here on.

We now construct an operator $\Sigma(k)$ which has $S_{\mathrm{red}}^{(k)}(\lambda)$ as its characteristic function. To this end let

$$
\begin{aligned}
& J(k)=\left[M\left(O_{-}\right) \vee M\left(O_{+}\right)\right] \ominus\left[E_{+}(k) \oplus E_{-}(k)\right], \quad k \in \mathbf{Z}_{+}, \\
& \Sigma(k)=P_{J(k)}^{K} U^{*} \mid J(k) .
\end{aligned}
$$

For $k=1,2,3, \ldots$ we have 


$$
\begin{aligned}
{\left[U^{*}-\Sigma(k)\right] J(k) } & =\left[I-P_{J(k)}^{K}\right] U^{*} J(k) \\
& =\left[I-P_{J(k)}^{K}\right] U^{*}\left\{M\left(O_{-}\right) \vee M\left(O_{+}\right) \ominus\left[E_{+}(k) \oplus E_{-}(k)\right]\right\} \\
& =\left[I-P_{J(k)}^{K}\right]\left\{M\left(O_{-}\right) \vee M\left(O_{+}\right) \ominus\left[E_{+}(k-1) \oplus E_{-}(k-1)\right]\right\} \\
& =O_{-}(k) .
\end{aligned}
$$

Similarly we can consider the adjoint case. We conclude that

$$
\begin{aligned}
{\left[U^{*}-\Sigma(k)\right](k) } & =O_{-}(k), \quad k=1,2, \ldots, \\
{\left[U-\Sigma(k)^{*}\right] J(k) } & =O_{+}(k), \quad k=1,2, \ldots
\end{aligned}
$$

By (2.19), (2.21), (2.41) we see that

$$
\left.P_{J(k)}^{K} U^{*} P_{E_{ \pm}}^{K}(k) U^{*}\right|_{J(k)}=0
$$

We also note that

$$
J(k) \oplus E_{+}(k) \oplus E_{-}(k)=M\left(O_{+}\right) \vee M\left(O_{-}\right)
$$

and is thus clearly a reducing subspace of $U$. For any $x \in M\left(O_{+}\right) \vee M\left(O_{-}\right)$we have

$$
P_{M\left(O_{-}\right) \vee M\left(O_{+}\right.}^{K} U^{*} x=U^{*} x
$$

Combining (2.42)-(2.44) we conclude

$$
\Sigma(k)^{n}=\left(\left.P_{J(k)}^{K} U^{*}\right|_{J(k)}\right)^{n}=\left.P_{J(k)}^{K} U^{* n}\right|_{J(k)}, \quad n, k=1,2, \ldots
$$

Now just as we defined in (1.30)-(1.33) and (1.20) the spaces $K, L, L$ *, $R$, and $R_{*}$ associated with the dilation $U$ of the arbitrary contraction $T$, we can consider their analogues denoted by $K(\Sigma(k)), L(\Sigma(k)), L_{*}(\Sigma(k))$, etc. for the contraction $\Sigma(k)$ defined on the Hilbert space $J(k)$. We note that (2.45) shows that $U^{*}$ on $K$, the minimal unitary dilation of $T^{*}$, is a unitary dilation for $\Sigma(k)$ if $k=1,2, \ldots$ By replacing $T$ by $\Sigma(k)$ and $U$ by $U^{*}$ in (1.30) and comparing this with (2.41) we conclude that for $k=1,2, \ldots$

$$
L(\Sigma(k))=O_{-}(k) ; \quad L_{*}(\Sigma(k))=O_{+}(k-1) .
$$

By applying (1.40) for $\Sigma(k)$ and $U^{*}$ instead of for the arbitrary contraction $T$ and its unitary dilation $U$ we see

$$
\begin{aligned}
K(\Sigma(k)) & =M_{-}\left(O_{+}(k)\right) \oplus J(k) \oplus M_{+}\left(O_{-}(k)\right), & & k=1,2,3, \ldots \\
& =E_{+}(k) \oplus J(k) \oplus E_{-}(k), & & k=1,2,3, \ldots
\end{aligned}
$$

Thus by (2.43) we see

$$
K(\Sigma(k))=M\left(O_{+}\right) \vee M\left(O_{-}\right), \quad k=1,2,3, \ldots
$$


Thus $\left.U^{*}\right|_{M\left(O_{+}\right) \vee M\left(O_{-}\right)}$is the minimal unitary dilation of $\Sigma(k)$ for $k=1,2$, .... Also from (1.40) we see

$$
\begin{aligned}
R(\Sigma(k)) & =K(\Sigma(k)) \ominus M\left(O_{+}\right), & k=1,2, \ldots, \\
R_{*}(\Sigma(k)) & =K(\Sigma(k)) \ominus M\left(O_{-}\right), & k=1,2, \ldots
\end{aligned}
$$

From (2.46) and (2.47) we see $K(\Sigma(k))=M(L(\Sigma(k))) \vee M\left(L_{*}(\Sigma(k))\right)$. We can conclude from Proposition II.1.4 of [9] that $\Sigma(k)$ is completely nonunitary for $k=1,2, \ldots$ In other words, there is no nonzero reducing subspace of $\Sigma(k)$ on which $\Sigma(k)$ is unitary.

We claim $\Sigma(k)$ has $S_{\text {red }}^{(k)}(\lambda)$ as its characteristic function. By Proposition 2.3 the characteristic function of $\Sigma(k)$ is given by the Fourier representation of $\left.P_{M\left(L_{*}(\Sigma(k))\right)}\right|_{M(L(\Sigma(k)))}$ with respect to $L_{*}(\Sigma(k))$ and $L(\Sigma(k))$. Using (2.46) we see that the characteristic function of $\Sigma(k)$ is given by the Fourier representation of

$$
P_{M\left(O_{+}\right)} \operatorname{lm}\left(O_{-}\right)
$$

with respect to $O_{+}(k-1)$ and $O_{-}(k), k=1,2, \ldots$ Denote this representation by

$$
\left\{O_{-}(k), O_{+}(k-1), \Theta_{\Sigma(k)}(\lambda)\right\}, \quad k=1,2, \ldots
$$

Comparing (2.49) and (2.50) with (2.31) and (2.32) we see

$$
S_{\text {red }}^{(k)}(\lambda)=\Theta_{\Sigma(k)}(\lambda), \quad|\lambda|<1,
$$

and so we have proven the following

THEOREM 2.6. The Fourier representation of the reduced scattering operator $S_{\mathrm{red}}^{(k)}$ with respect to $O_{-}(k)$ and $O_{+}(k-1)$ is the characteristic function of the completely nonunitary operator $\Sigma(k)$ for $k=1,2, \ldots$.

COROllary 2.7. For $k=1,2, \ldots$

$\left\{O_{+}(k-1), O_{-}(k), S_{\text {red }}^{(\tilde{k})}(\lambda)\right\}=\left\{O_{+}(k-1), O_{-}(k), \Theta_{\Sigma(k)}{ }^{*}(\lambda)\right\}$.

Proof. Apply (2.8) to (2.51) with the arbitrary contraction $T$ replaced by $\Sigma(k)$.

Before proceeding to the main result of this section we need to make some definitions. In [8] a major role is played by a local semigroup $Z(t)$ defined by

$$
Z(t)=P_{H \ominus D_{+}} T(t) P_{H \ominus D_{-}, \quad} t \geqslant 0 .
$$

That $Z(t)$ is a semigroup follows from the fact that in [8] (2.15) is satisfied. In analogy with (2.53) we define for $k \in \mathbf{Z}_{+}$

$$
Z^{(k)}(t)=P_{H \ominus E_{+}(k)}^{H} T(t) P_{H \ominus E_{-}(k),}^{H} \quad t \geqslant 0 .
$$


That $Z^{(k)}(t)$ is a semigroup follows from (2.20) and (2.21).

We prefer to work in the discrete framework. By defining $Z(k)$ as the cogenerator of $Z^{(k)}(t)$ and applying (1.18) we see

$$
Z(k)=P_{H \ominus E_{+}(k)}^{H} T P_{H \ominus E_{-}(k)}^{H}, \quad k \in \mathbf{Z} .
$$

The reader is cautioned that $Z(k)$ denotes a contraction operator as opposed to a semigroup. We may consider $Z(k)$ as an operator defined on $E_{0}(k)$ by noting that $Z(k) E_{ \pm}(k)=0$. We now prove some preliminary propositions about $Z(k)$.

Proposition 2.8. The minimal unitary dilation $U$ on $K$ for the contraction $T$ is a dilation of $Z(k)$ for $k \in Z_{+}$.

Proof. To verify the proposition it suffices to show

$$
Z(k)^{n}=\left.P_{E_{0}(k)}^{K} U^{n}\right|_{E_{0}(k)}, \quad n, k \in Z_{+} .
$$

To see this we note that

$$
\begin{aligned}
& Z(k)^{n} h=\left(P_{H \ominus E_{+}(k)}^{H} T P_{H \ominus E_{-}(k)}^{H}\right)^{n} h \quad \text { by }(2.55) \\
& =P_{H \ominus E_{+}(k)}^{H} T^{n} P_{H \ominus E_{-}(k)}^{H} \text { by (2.20) } \\
& =P_{H \ominus E_{+}(k)}^{H}\left(P_{H}^{K} U \eta_{H}\right)^{n} P_{H \ominus E_{-}(k)}^{H} \\
& =P_{H \ominus E_{+}(k)}^{H} P_{H}^{K} U\left(P_{H}^{K} U U_{H}\right)^{(n-1)} P_{H \ominus E_{-}(k)^{h}}^{h} \\
& =P_{H \ominus E_{+}(k)}^{K} U\left(\left.P_{H}^{K} U\right|_{H}\right)^{(n-1)} P_{H \ominus E_{-}(k)^{h}}^{h} \\
& =P_{H \ominus E_{+}(k)}^{K_{+}} U\left(P_{H}^{K_{+}} U \eta_{H}\right)^{(n-1)} P_{H \Theta E_{-}(k)^{h}}^{h}
\end{aligned}
$$

since $U^{n} H \subset K_{+}$. We note that by (1.32)

$$
P_{H}^{K^{+}}+P_{M_{+}^{+}(L)}^{K_{+}}=I_{K_{+}} .
$$

Thus

$$
\left.Z(k)^{n} h=P_{H \oplus E_{+}(k)}^{K_{+}} U\left(I-P_{M_{+}}^{K_{+}(L)}\right) U\right]^{(n-1)} P_{H \Theta E_{-}(k)}^{H} .
$$

Expand the right side of (2.57) by the binomial formula. Since

$$
P_{H \Theta E_{+}(k)}^{K_{+}} U P_{M_{+}^{+}(L)}^{K_{+}} U^{j}=0, \quad j \in Z_{+},
$$

we arrive at

$$
Z(k)^{n} h=P_{H \Theta E_{+}(k)}^{K_{+}} U^{n} P_{H \Theta E_{-}(k)}^{H}, \quad k, n \in Z_{+} .
$$

By restricting to $E_{0}(k)$ and noting that the above expression is unchanged if $K_{+}$ is replaced by $K$ we see that (2.56) holds. 
Proposition 2.9. $U$ on $K$ is the minimal unitary dilation of $Z(k)$ for $k$ $=1,2,3, \ldots$.

Note. Actually Proposition 2.9 is true for $k=0$ also but the proof is a little more involved. Since we do not need this fact, we omit the proof for this case.

Proof. To show $K$ is a minimal unitary dilation for $Z(k)$ we must show

$$
K=\bigvee_{n \in \mathbf{Z}} U^{n} E_{0}(k)
$$

Clearly it suffices to show $H \subset \bigvee_{n \in \mathbf{Z}} U^{n} E_{0}(k)$ since $\bigvee_{n \in Z} H^{n} H=K$. We claim

$$
E_{+}(k) \subset \bigvee_{n \in \mathbf{Z}_{+}} U^{n} E_{0}(k) ; \quad E_{-}(k) \subset \bigvee_{n \in \mathbf{Z}_{-}} U^{n} E_{0}(k) .
$$

Since $H=E_{+}(k) \oplus E_{0}(k) \oplus E_{-}(k)$ this would suffice to prove the proposition. To prove the first part of $(2.59)$ we note that, for $k \geqslant 1, O_{+}(k-1) \subset E_{0}(k)$. Now clearly

$$
\bigvee_{n \in \mathbf{Z}_{+}} U^{n} O_{+}(k-1) \supset E_{+}(k)
$$

so the first part of (2.59) is proven. The second part follows similarly.

Just as we defined $R(\Sigma(k)), L(\Sigma(k))$, and $L_{*}(\Sigma(k))$ in (1.20) and (1.30)(1.33) previously, we now denote the analogous subspaces for $Z(k)$ by $R(Z(k))$, $L(Z(k))$, and $L_{*}(Z(k))$.

Proposition 2.10. $R(Z(k)) \subset R \cap K_{+}(Z(k)), k \in \mathbf{Z}_{+}$.

Proof. Let $U_{+}$on $K_{+}$be the minimal isometric dilation of $T$ on $H$ and let $V_{+}$on $K(Z(k))$ be the minimal isometric dilation of $Z(k)$. We note that $\left.U_{+}\right|_{K_{+}(Z(k))}=V_{+}$. Then from Theorem III.2.1 of [9] we know

$$
\begin{aligned}
R & =\left\{x \in K_{+} \mid\left\|U_{+}^{* n} x\right\|=\|x\|, n \in Z_{+}\right\}, \\
R(Z(k)) & =\left\{x \in K_{+}(Z(k)) \mid\left\|V_{+}^{* n} x\right\|=\|x\|, n \in \mathbf{Z}_{+}\right\} .
\end{aligned}
$$

Since $K_{+}(Z(k))$ is invariant under $U_{+}, K \ominus K_{+}(Z(k))$ is invariant under $U_{+}^{*}$. Thus

$$
P_{K_{+}}^{K_{+}(Z(k))} U_{+}^{* n} \mid K_{+}(Z(k))=V_{+}^{* n} .
$$

Let $x \in R(Z(k))$. Then by $(2.60)$ we see

$$
\begin{aligned}
\|x\| & =\left\|V_{+}^{* n} x\right\|=\|\left. P_{K_{+}}^{K_{+}}(z(k)) U_{+}^{* n}\right|_{K_{+}(Z(k))^{x \|}} \\
& \leqslant \|\left. U_{+}^{* n}\right|_{K_{+}}(Z(k))^{x\|=\| U_{+}^{* n} x\|\leqslant\| x \| .}
\end{aligned}
$$

We conclude that $\|x\|=\left\|U_{+}^{* n} x\right\|$ for $n \in \mathbf{Z}_{+}$. Thus $x \in R$. Since $R(Z(k)) \subset$ $K_{+}(Z(k))$ the proposition follows. 
LeMma 2.11. For $k=1,2,3, \ldots$ the projection $P_{M\left(O_{-}\right)}^{K}$ defines a mapping from $L_{*}(Z(k))$ onto $U O_{-}(k)$ in the following manner:

$$
\begin{aligned}
& L_{*}(Z(k)) \stackrel{P_{M\left(O_{-}\right)}^{K}}{\longrightarrow} U O_{-}(k), \\
& U\left[U^{*}-Z(k)^{*}\right] e_{0}(k) \mapsto U\left[T^{*}-Z(k)^{*}\right] e_{0}(k)
\end{aligned}
$$

where $e_{0}(k) \in E_{0}(k)$.

Similarly the projection $P_{M\left(O_{+}\right)}^{K}$ defines a mapping from $L(Z(k))$ onto $O_{+}(k)$ as follows:

$$
\begin{aligned}
& L(Z(k)) \stackrel{P_{M\left(O_{+}\right)}^{K}}{\longrightarrow} O_{+}(k), \\
& {[U-Z(k)] e_{0}(k) \mapsto[T-Z(k)] e_{0}(k)}
\end{aligned}
$$

where $e_{0}(k) \in E_{0}(k)$.

Proof. To prove $(2.61)_{*}$ we make use of the following general proposition concerning orthogonal projections:

$$
p_{B}^{K} x=y \Longleftrightarrow(x-y) \perp B .
$$

Applying this to the case considered in $(2.61)_{*}$ we have $U\left[U^{*}-Z(k)^{*}\right] e_{0}(k)-$ $U\left[T^{*}-Z(k)^{*}\right] e_{0}(k)=U\left[U^{*}-T^{*}\right] e_{0}(k) \in L_{*}$. But by $(1.43) L_{*} \perp R$. By (1.38) and the adjoint of relation (1.15) applied to $E_{-}$we have

$$
\left\|P_{R}^{K_{+}} e_{-}\right\|=\lim _{n \rightarrow \infty}\left\|T^{* n} e_{-}\right\|=\left\|e_{-}\right\|
$$

for all $e_{-} \in E_{-}$. Thus $E_{-} \subset R$ and so

$$
M\left(O_{-}\right)=M\left(E_{-}\right) \subset M(R)=R .
$$

We conclude that $L_{*} \perp M\left(O_{-}\right)$and a fortiori that $U\left(U^{*}-T^{*}\right) e_{0}(k) \perp M\left(O_{-}\right)$. Thus $(2.61)_{*}$ holds. To show that the mapping is onto $U O_{-}(k)$ we note that

$$
\begin{aligned}
{\left[T^{*}-Z(k)^{*}\right] e_{0}(k) } & =\left[P_{H} U^{*}-P_{E_{0}(k)} U^{*}\right] e_{0}(k) \\
& =P_{E_{-}(k) \oplus E_{+}(k)} U^{*} e_{0}(k) .
\end{aligned}
$$

Since $U E_{+}(k) \subset E_{+}(k)$ and $E_{0}(k) \perp E_{+}(k)$ we see that

$$
\left[T^{*}-Z(k)^{*}\right] E_{0}(k)=P_{E_{-}(k)} U^{*} E_{0}(k) .
$$

For $k=1,2,3, \ldots$ we have $\left[E_{0}(k) \cap E_{-}(k-1)\right]=O_{-}(k-1)$. Since $U^{*}$ acts as a bilateral shift on $M\left(O_{-}\right), U^{*} O_{-}(k-1)=O_{-}(k)$. Thus substituting into (2.64) we have 


$$
\begin{aligned}
U\left[T^{*}-Z(k)^{*}\right] E_{0}(k) & \supseteq U P_{E_{-}(k)} U^{*}\left[E_{0}(k) \cap E_{-}(k-1)\right] \\
& \supseteq U P_{E_{-}(k)} U^{*} O_{-}(k-1) \supseteq U P_{E_{-}(k)} O_{-}(k)=U O_{-}(k)
\end{aligned}
$$

and the mapping defined in $(2.61)_{*}$ is onto.

The adjoint case considered in (2.61) is proven similarly.

Lemma 2.12. For $k=1,2,3, \ldots$ the projection $P_{M\left(L_{*}\right)}^{K}$ defines a mapping from $L_{*}(Z(k))$ to $L_{*}$ given by

$$
\begin{aligned}
& L_{*}(Z(k)) \stackrel{P_{M\left(L_{*}\right)}^{K}}{\longmapsto} L_{* \cdot} \\
& U\left[U^{*}-Z(k)^{*}\right] e_{0}(k) \longmapsto U\left[U^{*}-T^{*}\right] e_{0}(k)
\end{aligned}
$$

where $e_{0}(k) \in E_{0}(k)$.

Similarly the projection $P_{M(L)}^{K}$ defines a mapping from $L(Z(k))$ to $L$ given by

$$
\begin{aligned}
& L(Z(k)) \stackrel{P_{M(L)}^{K}}{\longrightarrow} L, \\
& {[U-Z(k)] e_{0}(k) \mapsto[U-T] e_{0}(k)}
\end{aligned}
$$

where $e_{0}(k) \in E_{0}(k)$.

Proof. Again using (2.62) we see

$$
\begin{aligned}
U\left(U^{*}-Z(k)^{*}\right) e_{0}(k)-U\left(U^{*}-T^{*}\right) e_{0}(k) & =U\left(T^{*}-Z(k)^{*}\right) e_{0}(k) \\
=U\left(T^{*}-P_{H \ominus E_{-}(k)} T^{*}\right) e_{0}(k) & =U P_{E_{-}(k)} T^{*} e_{0}(k) \in M\left(E_{-}\right)=M\left(O_{-}\right) .
\end{aligned}
$$

By (2.63) we see that $M\left(O_{-}\right) \subset R$. Since $R \perp M\left(L_{*}\right)$ by (1.40) we conclude that

$$
U\left(T^{*}-Z(k)^{*}\right) e_{0}(k) \perp M\left(L_{*}\right) \text {. }
$$

Thus $(2.70)_{*}$ holds.

The adjoint case considered in (2.65) in proven similarly.

We now come to the main result of this section.

THEOREM 2.13. For $k=1,2, \ldots$ and $|\lambda|<1$ let

$$
\begin{gathered}
\left\{L(Z(k)), L_{*}(Z(k)), \Theta_{Z(k)}(\lambda)\right\}, \\
\left\{O_{+}(k), O_{-}(k), \Theta_{\Sigma(k) *}(\lambda)\right\}, \\
\left\{L, L_{*}, \Theta_{T}(\lambda)\right\}
\end{gathered}
$$

denote the characteristic functions of $Z(k), \Sigma(k)^{*}$, and $T$ respectively. Then there exist analytic (contraction) operator-valued functions

$$
\left\{O_{+}(k), L_{*}, \Omega_{21}^{(k)}(\lambda)\right\}
$$




$$
\left\{L, O_{-}(k), \Omega_{12}^{(k)}(\lambda)\right\}
$$

such that for any $e_{0}(k) \in E_{0}(k)$

$$
\begin{aligned}
\Theta_{Z(k)}(\lambda)[U-Z(k)] e_{0}(k) & \\
= & \left(\begin{array}{cc}
\Theta_{\Sigma(k) *}(\lambda) & \Omega_{12}^{(k)}(\lambda) \\
\Omega_{21}^{(k)}(\lambda) & \Theta_{T}(\lambda)
\end{array}\right)\left(\begin{array}{c}
{[T-Z(k)] e_{0}(k)} \\
{[U-T] e_{0}(k)}
\end{array}\right) .
\end{aligned}
$$

Proof. Let

$$
\begin{aligned}
\Theta_{Z(k)}(\lambda) & =\sum_{n=0}^{\infty} \lambda^{n} \Theta_{Z(k), n}, & & |\lambda|<1, \\
\Theta_{\Sigma(k)^{*}}(\lambda) & =\sum_{n=0}^{\infty} \lambda^{n} \Theta_{\Sigma(k)^{*}, n}, & & |\lambda|<1, \\
\Theta_{T}(\lambda) & =\sum_{n=0}^{\infty} \lambda^{n} \Theta_{T, n}, & & |\lambda|<1 .
\end{aligned}
$$

To prove the theorem it clearly suffices to show that there exist contractions $\Omega_{12, n}^{(k)}$ and $\Omega_{21, n}^{(k)}$ such that

$$
\Theta_{Z(k), n}=\left(\begin{array}{ll}
\Theta_{\Sigma(k)^{*}, n} & \Omega_{21}^{(k)}(\lambda) \\
\Omega_{21, n}^{(k)} & \Theta_{T}(\lambda)
\end{array}\right), \quad n=0,1, \ldots
$$

Before proceeding with the proof we describe the overall plan of attack. The idea of the proof is to decompose the domain of $\Theta_{Z(k), m}$ into two orthogonal subspaces which are the domains of $\Theta_{\Sigma(k)^{*}, m}$ and $\Theta_{T, m}$ respectively. With this decomposition we prove that (2.72) holds for any vector in either the domain of $\Theta_{\Sigma(k)^{*}, m}$ or the domain of $\Theta_{T, m}$. Linearity then implies the result.

With the help of Lemma 2.11 and (2.15)-(2.17) (applied to $Z(k)$ ), we can construct the following diagrams for $m=1,2,3, \ldots$.

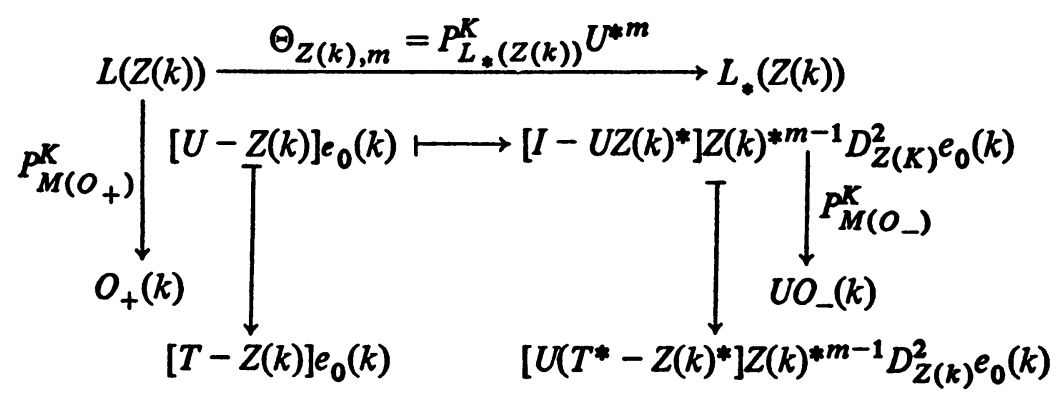




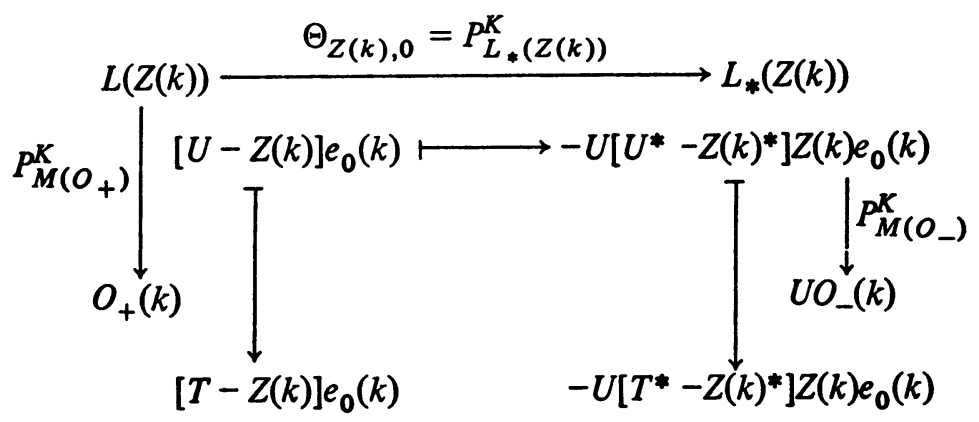

By Lemma $2.11 P_{M\left(O_{-}\right)}^{K}$ defines a mapping from $L_{*}$ to $U O_{-}(k)$. The right-hand side assertion of (2.73) is therefore a consequence of $(2.61)_{*}$ with $e_{0}(k)$ replaced by $Z(k)^{* m-1} D_{Z(k)}^{2} e_{0}(k)$. Similarly to check the mapping by $P_{M\left(O_{-}\right)}^{K}$ in (2.74) we note that this is just $(2.61)_{*}$ with $e_{0}(k)$ replaced by $Z(k) e_{0}(k)$.

Before proceeding we prove two important identities. First we claim that

$$
P_{U O_{-}(k)}^{K} U^{* m}(U-Z(k)) e_{0}(k)=U\left(T^{*}-Z(k)^{*}\right) Z(k)^{* m-1} D_{Z(k)}^{2} e_{0}(k) .
$$

To verify this we note

$$
\begin{aligned}
\left(U^{* m}(U-Z(k)) e_{0}(k)-U\left(T^{*}-Z(k)^{*}\right) Z(k)^{* m-1} D_{Z(k)}^{2} e_{0}(k), U O_{-}(k)\right) \\
=\left(\left[U^{* m}-U^{* m+1} Z(k)-T^{*} Z(k)^{* m-1} D_{Z(k)}^{2}\right.\right. \\
\left.\left.\quad+Z(k)^{*} Z(k)^{* m-1} D_{Z(k)}^{2}\right] e_{0}(k), O_{-}(k)\right) \\
=\left(\left[U^{* m}-U^{* m+1} Z(k)-T^{*} Z(k)^{* m-1}+T^{*} Z(k)^{* m} Z(k)\right.\right. \\
\left.\left.+Z(k)^{* m}-Z(k)^{* m+1} Z(k)\right] e_{0}(k), O_{-}(k)\right) \\
=\left(\left[U^{* m}-U^{* m+1} Z(k)-T^{*} Z(k)^{* m-1}\right.\right. \\
\left.\left.\quad+T^{*} Z(k)^{* m} Z(k)\right] e_{0}(k), O_{-}(k)\right) \\
=\left(\left[U^{* m}-T^{*} Z(k)^{* m-1}\right] e_{0}(k)\right. \\
\left.\quad+\left[T^{*} Z(k)^{* m} Z(k)-U^{* m+1} Z(k)\right] e_{0}(k), O_{-}(k)\right) \\
=\left(\left[T^{* m}-T^{*} Z(k)^{* m-1}\right] e_{0}(k)\right. \\
\left.\quad+\left[T^{*} Z(k)^{* m} Z(k)-T^{* m+1} Z(k)\right] e_{0}(k), O_{-}(k)\right) \\
=\left(\left[T^{* m-1}-Z(k)^{* m-1}\right] e_{0}(k)+\left[Z(k)^{* m}-T^{* m}\right] Z(k) e_{0}(k), T O_{-}(k)\right) .
\end{aligned}
$$

Now the right-hand side of this inner product is in $E_{0}(k)$ while the left-hand side is an element of $E_{-}(k)$. By (2.25) we conclude (2.75).

Second we claim that 


$$
\left.P_{U O_{-}(k)}^{K}[U-Z(k)] e_{0}(k)=-U T^{*}-Z(k) *\right] Z(k) e_{0}(k) .
$$

To verify this we note that

$$
\begin{aligned}
\left([U-Z(k)] e_{0}(k)+U\left[T^{*}-Z(k)^{*}\right] Z(k) e_{0}(k), U O_{-}(k)\right) \\
=\left(\left[I-U^{*} Z(k)+T^{*} Z(k)-Z(k) * Z(k)\right] e_{0}(k), O_{-}(k)\right) \\
=\left(\left[I-U^{*} Z(k)+T^{*} Z(k)\right] e_{0}(k), O_{-}(k)\right) \\
=\left(e_{0}(k), O_{-}(k)\right)-\left(\left[U^{*}-T^{*}\right] Z(k) e_{0}(k), O_{-}(k)\right) .
\end{aligned}
$$

By (2.26) the first term is zero and by (1.40) the second term is zero. We will use (2.75) and (2.76) later in the proof.

As noted in Lemma 2.11, $M\left(O_{-}\right) \subset R$. By (1.40), (2.61), and (2.65) we see that for any $e_{0}(k) \in E_{0}(k)$ and $k \in \mathbf{Z}_{+}$

$$
[U-Z(k)] e_{0}(k)=[U-T] e_{0}(k) \oplus[T-Z(k)] e_{0}(k) .
$$

This decomposes $L(Z(k))$ into orthogonal vectors in $L$ and $O_{+}(k)$.

Now suppose that we restrict our attention to those $e_{0}(k) \in E_{0}(k)$ whose first component in (2.77) is zero. Then we have

$$
[U-Z(k)] e_{0}(k)=[T-Z(k)] e_{0}(k) .
$$

Then substituting (2.78) into the left-hand side of (2.75) we conclude

$$
P_{U O_{-}(k)}^{K} U^{* m}(T-Z(k)) e_{0}(k)=U\left(T^{*}-Z(k)^{*}\right) Z(k)^{* m-1} D_{Z(k)}^{2} e_{0}(k) \text {. }
$$

Recalling (2.46) we see

$$
L_{*}\left(\Sigma(k)^{*}\right)=U O_{-}(k) ; \quad L\left(\Sigma(k)^{*}\right)=O_{+}(k) .
$$

Thus we conclude

$$
\begin{aligned}
& \left.P_{L_{*}\left(\Sigma(k)^{*}\right)} U^{* m}\right|_{L\left(\Sigma(k)^{*}\right)}[T-Z(k)] e_{0}(k) \\
& =U\left(T^{*}-Z(k)^{*}\right) Z(k)^{* m-1} D_{Z(k)}^{2} e_{0}(k) .
\end{aligned}
$$

By (2.16) applied to $\Sigma(k)^{*}$ we see that for $m=1,2, \ldots$

(2.81) $\Theta_{\Sigma(k)^{*}, m}[T-Z(k)] e_{0}(k)=U\left(T^{*}-Z(k)^{*}\right) Z(k)^{* m-1} D_{Z(k)}^{2} e_{0}(k)$.

Substituting (2.78) into the left-hand side of (2.76) we conclude

$$
P_{U O_{-}(k)}^{K}[T-Z(k)] e_{0}(k)=-U\left(T^{*}-Z(k)^{*}\right) Z(k) e_{0}(k) .
$$

Again by $(2.46)^{*}$ since $[T-Z(k)] E_{0}(k)=O_{+}(k)$ we see

(2.83) $\left.P_{L_{*}\left(\Sigma(k)^{*}\right)}^{K}\right|_{L\left(\Sigma(k)^{*}\right)}[T-Z(k)] e_{0}(k)=-U\left(T^{*}-Z(k)^{*}\right) Z(k) e_{0}(k)$.

By $(2.16)$ applied to $\Sigma(k) *$ we see 


$$
\Theta_{\Sigma(k), 0}[T-Z(k)] e_{0}(k)=-U\left(T^{*}-Z(k)^{*}\right) Z(k) e_{0}(k) .
$$

We now define for all $e_{0}(k)$ satisfying (2.78) and $n \in Z_{+}$

$$
\Omega_{21, n}^{(k)}[T-Z(k)] e_{0}(k)=P_{L} \theta_{Z(k), n}[U-Z(k)] e_{0}(k) .
$$

We claim

$\Omega_{21, n}^{(k)}[T-Z(k)] e_{0}(k)= \begin{cases}U\left(U^{*}-T^{*}\right) Z(k)^{* n-1} D_{Z(k)}^{2} e_{0}(k), & n=1,2, \ldots, \\ -U\left(U^{*}-T^{*}\right) Z(k) e_{0}(k), & n=0 .\end{cases}$

To see this it suffices to show

$$
\begin{aligned}
& \left(U\left(U^{*}-Z(k)^{*}\right) Z(k)^{* m-1} D_{Z(k)}^{2} e_{0}(k)\right. \\
& \left.-U\left(U^{*}-T^{*}\right) Z(k)^{* m-1} D_{Z(k)}^{2} e_{0}(k), L_{*}\right)=0
\end{aligned}
$$

and

$$
\left(-U\left(U^{*}-Z(k) *\right) Z(k) e_{0}(k)+U\left(U^{*}-T^{*}\right) Z(k) e_{0}(k), L_{*}\right)=0
$$

We omit this simple calculation.

We now proceed to the second column of the matrix in (2.72). It is easy to check the validity of the following diagrams:
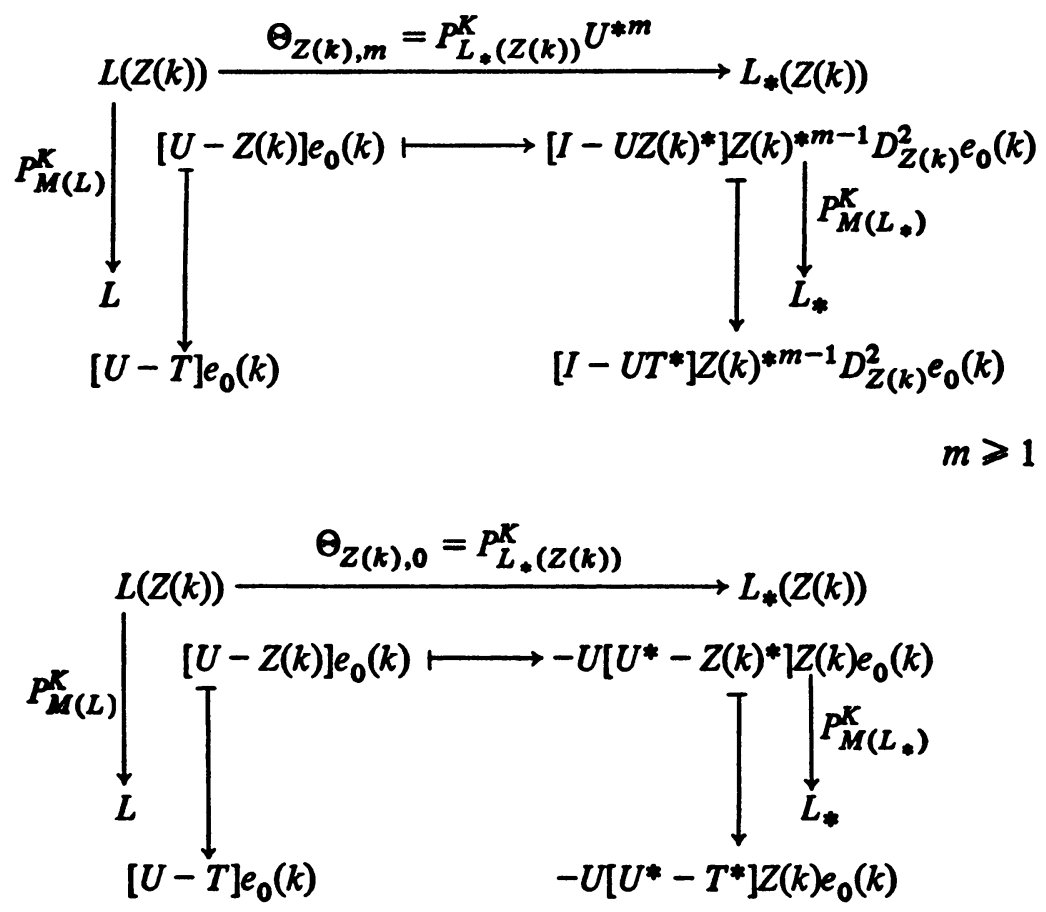

For example to check the mapping by $P_{M\left(L_{*}\right)}^{K}$ in (2.85) we note that this is just $(2.65)_{*}$ with $e_{0}(k)$ replaced by $Z(k)^{* m-1} D_{Z(k)}^{2} e_{0}(k)$. Similarly to check 
the mapping by $P_{M\left(L_{*}\right)}^{K}$ in (2.86) we note that this is just $(2.65)_{*}$ with $e_{0}(k)$ replaced by $Z(k) e_{0}(k)$.

We claim

and

$$
\begin{aligned}
P_{L}^{K} U^{* m}(U-Z(k)) e_{0}(k)=\left[I-U T^{*}\right] Z(k)^{* m-1} D_{Z(k)}^{2} e_{0}(k), & \\
m & =1,2, \ldots,
\end{aligned}
$$

$$
P_{L_{*}}^{K}[U-Z(k)] e_{0}(k)=-U\left[U^{*}-T^{*}\right] Z(k)^{*} e_{0}(k) .
$$

The calculations are similar to those done for column one and are omitted.

We now limit our attention to those $e_{0}(k) \in E_{0}(k)$ whose second component in (2.77) is zero. For these $e_{0}(k)$ 's we have

$$
[U-Z(k)] e_{0}(k)=[U-T] e_{0}(k) .
$$

Substituting (2.89) into the left-hand side of (2.87) we conclude

$$
P_{L_{*}}^{K} U^{* m}[U-T] e_{0}(k)=\left[I-U T^{*}\right] Z(k)^{* m-1} D_{Z(k)}^{2} e_{0}(k) .
$$

By (2.16) we see

$$
\Theta_{T, m}[U-T] e_{0}(k)=\left[I-U T^{*}\right] Z(k)^{* m-1} D_{Z(k)}^{2} e_{0}(k) .
$$

Substituting (2.89) into the left-hand side of (2.88) we see by (2.16) that

$$
\Theta_{T, 0}[U-T] e_{0}(k)=-U\left[U^{*}-T^{*}\right] Z(k) * e_{0}(k) .
$$

So far we have shown that the theorem holds for those $e_{0}(k) \in E_{0}(k)$ which are of a special form. In particular we have shown that the theorem holds for those $e_{0}(k)$ which cause the first or second component of (2.77) to be zero. We now piece together these results and conclude that the theorem holds for all $e_{0}(k) \in E_{0}(k)$.

We decompose $E_{0}(k)$ for $k=1,2,3, \ldots$ as follows

$$
E_{0}(k)=E_{0}(k-1) \oplus\left[E_{0}(k) \ominus E_{0}(k-1)\right]
$$

This allows us to write

$$
e_{0}(k)=e_{0}^{\prime}(k) \oplus e_{0}^{\prime \prime}(k)
$$

where $e_{0}^{\prime}(k) \in E_{0}(k-1)$ and $e_{0}^{\prime \prime}(k) \in E_{0}(k) \ominus E_{0}(k-1)$. We claim $e_{0}^{\prime \prime}(k)$ satisfies (2.83) and $e_{0}^{\prime}(k)$ satisfies (2.94). To see this we note that

$$
e_{0}^{\prime \prime}(k) \in E_{0}(k) \ominus E_{0}(k-1) \subset E_{+}(k-1) \oplus E_{-}(k-1) \text {. }
$$

For $k=2,3, \ldots$ the actions of $U$ and $T$ on $E_{ \pm}(k-1)$ coincide; thus (2.78) is satisfied by $e_{0}^{\prime \prime}(k)$. With regard to $e_{0}^{\prime}(k)$ we note that for $k=1,2, \ldots$ 


$$
T E_{0}(k-1) \subset E_{0}(k)
$$

and so the actions of $T$ and $Z$ coincide on $E_{0}(k-1)$. Thus $e_{0}^{\prime}(k)$ satisfies (2.89).

Since

$$
\Theta_{Z(k), n}[U-Z(k)] e_{0}(k)=\Theta_{Z(k), n}[U-Z(k)] e_{0}^{\prime}(k)+\Theta_{Z(k), n}[U-Z(k)] e_{0}^{\prime \prime}(k)
$$

we can use the linearity of (2.72) to conclude the theorem. We note that (2.89) appears to imply that $\Theta_{T, m}$ depends on $k$. This is not so since $(U-T) E_{ \pm}(k)=0$ for $k=1,2, \ldots$. Finally we observe that (2.84) and (2.91) imply that $\Omega_{i j}^{(k)}(\lambda), i, j=1,2 ; i \neq j$, are contraction-valued functions as claimed.

Corollary 2.14. For $|\lambda|<1, k=2,3, \ldots$, and $e_{0}(k) \in E_{0}(k)$

$$
\begin{aligned}
& \Theta_{Z(k)}(\lambda)[U-Z(k)] e_{0}(k) \\
&= \Theta_{\Sigma(k) *}(\lambda)[T-Z(k)] e_{0}(k) \oplus \Theta_{T}(\lambda)[U-T] e_{0}(k) \\
&=\left.U T^{*}-Z(k)^{*}\right]\left[I-\lambda Z(k)^{*}\right]^{-1}[\lambda-Z(k)] e_{0}(k) \\
& \oplus U\left[U^{*}-T^{*}\right]\left[I-\lambda Z(k)^{*}\right]^{-1}[\lambda-Z(k)] e_{0}(k) .
\end{aligned}
$$

Proof. By (2.93) we see

$$
\begin{aligned}
\Theta_{Z(k), 0}[U-Z(k)] e_{0}(k) & =\Theta_{Z(k), 0}[U-Z(k)]\left(e_{0}^{\prime}(k)+e_{0}^{\prime \prime}(k)\right) \\
& =\left(\begin{array}{cc}
\Theta_{\Sigma(k)^{*}, 0} & \Omega_{12,0}^{(k)} \\
\Omega_{21,0}^{(k)} & \Theta_{T, 0}
\end{array}\right)\left(\begin{array}{c}
{[T-Z(k)] e_{0}^{\prime \prime}(k)} \\
{[U-T] e_{0}^{\prime}(k)}
\end{array}\right) \\
& =\left(\begin{array}{c}
-U\left(T^{*}-Z(k)^{*}\right) Z(k) e_{0}(k) \\
-U\left(U^{*}-T^{*}\right) Z(k) e_{0}(k)
\end{array}\right) .
\end{aligned}
$$

For $n=1,2,3, \ldots$ we have

$$
\begin{aligned}
\Theta_{Z(k), n}[U-Z(k)] e_{0}(k) & =\Theta_{Z(k), n}[U-Z(k)]\left(e_{0}^{\prime}(k)+e_{0}^{\prime \prime}(k)\right) \\
& =\left(\begin{array}{cc}
\Theta_{\Sigma(k)^{*}, n} & \Omega_{12, n}^{(k)} \\
\Omega_{21, n}^{(k)} & \Theta_{T, n}
\end{array}\right)\left(\begin{array}{c}
{[T-Z(k)] e_{0}^{\prime \prime}(k)} \\
{[U-T] e^{\prime}(k)}
\end{array}\right) \\
& =\left(\begin{array}{c}
U\left(T^{*}-Z(k)^{*}\right) Z(k)^{* n-1} D_{Z(k)}^{2} e_{0}(k) \\
U\left(U^{*}-T^{*}\right) Z(k)^{* n-1} D_{Z(k)}^{2} e_{0}(k)
\end{array}\right) .
\end{aligned}
$$

Thus a simple calculation shows 


$$
\begin{aligned}
\Theta_{Z(k)}(\lambda)[U-Z(k)] e_{0}(k)=\sum_{n=0}^{\infty} \lambda^{n} \Theta_{Z(k), n}[U-Z(k)] e_{0}(k) \\
= \\
\left..9 \begin{array}{c}
U\left(T^{*}-Z(k)^{*}\right)\left[-Z(k)+\lambda\left(I-\lambda Z(k)^{*}\right)^{-1} D_{Z(k)}^{2}\right] e_{0}(k) \\
U\left(U^{*}-T^{*}\right)\left[-Z(k)+\lambda\left(I-\lambda Z(k)^{*}\right)^{-1} D_{Z(k)}^{2}\right] e_{0}(k)
\end{array}\right) \\
=\left(\begin{array}{c}
U\left(T^{*}-Z(k)^{*}\right)\left(I-\lambda Z(k)^{*}\right)^{-1}(\lambda-Z(k)) e_{0}(k) \\
U\left(U^{*}-T^{*}\right)\left(I-\lambda Z(k)^{*}\right)^{-1}(\lambda-Z(k)) e_{0}(k)
\end{array}\right)
\end{aligned}
$$

From the calculation in (2.95) we see

$$
\Theta_{\Sigma(k) *}(\lambda)[T-Z(k)] e_{0}(k)=\Omega_{12}^{(k)}(\lambda)[U-T] e_{0}(k)
$$

and

$$
\Theta_{T}(\lambda)[U-T] e_{0}(k)=\Omega_{21}^{(k)}(\lambda)[T-Z(k)] e_{0}(k) .
$$

Thus we can identify each row of (2.96) as

$$
\left(\begin{array}{c}
\Theta_{\Sigma(k)}(\lambda)[T-Z(k)] e_{0}(k) \\
\Theta_{T}(\lambda)[U-T] e_{0}(k)
\end{array}\right)
$$

The corollary now follows by noting that the columns of (2.96) are orthogonal.

$$
\text { Corollary 2.15. For }|\lambda|<1, k=2,3, \ldots \text {, and } e_{0}(k) \in E_{0}(k)
$$

$$
\begin{aligned}
\Theta_{Z(k)}(\lambda)[U & -Z(k)] e_{0}(k) \\
& =S_{\mathrm{red}}^{(\tilde{k})}(\lambda)[T-Z(k)] e_{0}(k) \oplus \Theta_{T}(\lambda)[U-T] e_{0}(k) .
\end{aligned}
$$

Proof. Apply Corollary 2.7 to Corollary 2.14.

LEMMA 2.16. For $k \in \mathrm{Z}_{+}$and $e_{0}(k) \in E_{0}(k)$

$$
\left\|\left(U-\Sigma(k)^{*}\right) e_{0}(k)\right\|=\left\|(T-Z(k)) e_{0}(k)\right\| .
$$

Proof.

$$
\begin{aligned}
\left\|\left(U-\Sigma(k)^{*}\right) e_{0}(k)\right\|^{2}=\left\|P_{E_{+}(k) \oplus E_{-}(k)} U e_{0}(k)\right\|^{2} \\
\quad=\left\|P_{E_{+}(k)} U e_{0}(k)\right\|^{2}=\left(P_{E_{+}(k)} U e_{0}(k), T e_{0}(k)\right) \\
=\left\|P_{E_{+}(k)} T e_{0}(k)\right\|^{2}=\left\|(T-Z(k)) e_{0}(k)\right\|^{2}
\end{aligned}
$$

Corollary 2.17. For $|\lambda|<1, k=2,3, \ldots$, and $e_{0}(k) \in E_{0}(k)$

$$
\begin{aligned}
& \Theta_{Z(k)}(\lambda)[U-Z(k)] e_{0}(k) \\
& \quad=\Theta_{\Sigma(k) *}(\lambda)\left[U-\Sigma(k)^{*}\right] e_{0}(k) \oplus \Theta_{T}(\lambda)[U-T] e_{0}(k) .
\end{aligned}
$$


Proof. Use Lemma 2.16 and the fact that characteristic functions are defined only up to unitary equivalence.

Finally by considering the dual system generated by $T *$ we obtain the following

CoRollary 2.18. For $|\lambda|<1, k=2,3, \ldots$ and $e_{0}(k) \in E_{0}(k)$

$$
\begin{aligned}
\Theta_{Z(k)} & (\lambda)\left[U^{*}-Z(k)^{*}\right] e_{0}(k) \\
= & S_{\mathrm{red}}^{(k)}(\lambda)\left[T^{*}-Z(k)^{*}\right] e_{0}(k) \oplus \Theta_{T^{*}}(\lambda)\left[U^{*}-T^{*}\right] e_{0}(k) \\
= & U^{*}(T-Z(k))(I-\lambda Z(k))^{-1}\left(\lambda-Z(k)^{*}\right) e_{0}(k) \\
& \oplus U^{*}(U-T)(I-\lambda Z(k))^{-1}\left(\lambda-Z(k)^{*}\right) e_{0}(k) .
\end{aligned}
$$

REMARK. In Theorem 2.15 if various (rather strong) assumptions are placed on $\Theta_{z(k)}(\lambda)$ we can conclude that there exists a meromorphic operatorvalued function, $\Omega(\lambda)$, defined in the exterior of the unit disc, so that the radial limits of $\widetilde{S}_{\mathrm{red}}^{(k)}(\lambda)$ and $\Omega(\lambda)$ agree almost everywhere on the unit circle. If this is the case $\widetilde{S}_{\text {red }}^{(k)}(\lambda)$ is said to have a meromorphic pseudo-analytic extension to the exterior of the unit disc. See [4] for details; we mention here only that one sufficient asumption on $\Theta_{z(k)}(\lambda)$ is that it be matrix-valued.

We now make an additional assumption on the $E_{ \pm}$. We assume

$$
M\left(E_{ \pm}\right)=M\left(D_{ \pm}\right) .
$$

This assumption is satisfied by the systems which Lax and Phillips consider. To see this we prove that $(2.100)$ is equivalent to the following (equivalent) statements:

$$
\begin{gathered}
\operatorname{s-lim}_{n \rightarrow \infty} Z(k)^{n}=0=\underset{n \rightarrow \infty}{s-\lim _{n \rightarrow \infty} Z(k)^{* n},} \\
s-\lim _{t \rightarrow \infty} Z^{(k)}(t)=0=s-\lim _{t \rightarrow \infty} Z^{(k)}(t)^{*} .
\end{gathered}
$$

Proposition 2.19. We have the following equivalences:

$$
(2.100) \Longleftrightarrow(2.101) \Longleftrightarrow(2.101)_{c t s} \text {. }
$$

Proof. The equivalence of (2.101) and (2.101) cts $_{\text {is }}$ just Proposition III.9.1 of [9]. To see the first equivalence we note that (2.101) holds if and only if for all $h \in H$

$$
\operatorname{sim}_{n \rightarrow \infty} P_{H \Theta E_{+}}^{H} T^{n} h=0=\operatorname{s-lim}_{n \rightarrow \infty} P_{H \ominus E_{-}}^{H} T^{* n} h .
$$

By Lemma 1.3 applied to $E_{ \pm}$and $U^{ \pm 1}$ instead of $D_{*}$ and $U^{*}$ we see that (2.102) holds if and only if $R=M\left(E_{-}\right), R_{*}=M\left(E_{+}\right)$. But $R=H_{-}=M\left(D_{-}\right)$and $R_{*}=$ 
$H_{+}=M\left(D_{+}\right)$by Lemma 1.3 and the energy decay assumptions for $D_{ \pm}$, i.e.

(1.25). Thus $M\left(D_{ \pm}\right)=M\left(E_{ \pm}\right)$as required.

We note that (2.101) implies (by Theorem II.1.2 of [9]) that $R(Z(k))=$ $R_{*}(Z(k))=\{0\}$. This result is stronger than Proposition 2.10, but of course we need to assume more-namely (2.100).

We next find another equivalent form of (2.101).

Proposition 2.20. $\left\{L(Z), L_{*}(Z), \Theta_{Z}(\lambda)\right\}$ is inner if and only if $\lim _{n \rightarrow \infty} Z^{* n} h$ $=0$ for all $h \in H$.

$\left\{L(Z), L_{*}(Z), \Theta_{Z}(\lambda)\right\}$ is *-inner if and only if $\lim _{n \rightarrow \infty} Z^{n} h=0$ for all $h \in H$.

ProOF. See Proposition VI.3.5 of [9].

Corollary 2.21. For $k=1,2, \ldots \Theta_{Z(k)}(\lambda)$ is inner and *-inner.

Proof. By assumption (2.100) holds. Proposition 2.19 implies that (2.101) holds. Proposition 2.20, applied to $Z(k)$, implies the corollary.

REMARK. By (2.100) it is clear that $\alpha$ and $\beta$ defined by (2.29) are unitary maps. Using the same localization procedure as in Claim 2.5, we can show $\alpha\left(e^{i t}\right)$ and $\beta\left(e^{i t}\right)$ are unitary almost everywhere on the unit circle.

3. The location of the poles and zeroes of $S_{\text {red }}$. In this section we apply Theorem 2.13 and its corollaries and derive discrete versions of Theorems 2 and 3 found in the Introduction. The continuous analogue is proven in \$4. Before proceeding we need two technical lemmas which allow us to relate $\Theta_{z(k)}(\lambda)$, $k=0,1,2, \ldots$ We denote $Z(0)$ by $Z, E_{0}(0)$ by $E_{0}, O_{ \pm}(0)$ by $O_{ \pm}$, etc.

LEMMA 3.1. Let $0<|\lambda|<1$ and $k=0,1,2, \ldots$ Then $\Theta_{Z(k)}(\lambda)$ is an isomorphism (i.e. one-to-one, onto, and bicontinuous) if and only if $\Theta_{z}(\lambda)$ is an isomorphism.

ProOF. Corresponding to the decomposition

$$
E_{0}(k)=F_{-}(k) \oplus E_{0} \oplus F_{+}(k)
$$

where

$$
F_{-}(k)=\bigoplus_{j=0}^{k-1} O_{-}(j), \quad F_{+}(k)=\bigoplus_{j=0}^{k-1} O_{+}(j)
$$

we have a decomposition for $Z(k)$ as

$$
Z(k)=\left(\begin{array}{ccc}
Z_{11} & * & * \\
0 & Z & * \\
0 & 0 & Z_{33}
\end{array}\right)
$$

where 


$$
\begin{aligned}
Z_{11}: & F_{-}(k) \rightarrow F_{-}(k), \quad k=0,1,2, \ldots, \\
Z: E_{0} & \rightarrow E_{0}, \\
Z_{33}: & F_{+}(k) \rightarrow F_{+}(k), \quad k=0,1,2, \ldots
\end{aligned}
$$

The stars denote contraction operators and the zeroes are due to the invariance of the subspaces in (3.1) under $Z(k)$.

Let $\Theta_{Z(k)}(\lambda), \Theta_{Z}(\lambda), \Omega_{+}^{(k)}(\lambda), \Omega_{-}^{(k)}(\lambda)$ denote the characteristic functions of $Z(k), Z, Z_{33}$, and $Z_{11}$ respectively. Then by applying Theorem VII.2.1 of [9] to

$$
\left.\left(\begin{array}{cc}
Z_{11} & * \\
0 & Z
\end{array}\right) * \begin{array}{c}
* \\
0
\end{array} Z_{33}\right) \text { and }\left(\begin{array}{cc}
Z_{11} & * \\
0 & Z
\end{array}\right)
$$

successively we obtain the following factorization of $\Theta_{Z(k)}(\lambda)$ :

$$
\Theta_{Z(k)}(\lambda)=\Theta_{33}^{(k)}(\lambda) \Theta_{22}^{(k)}(\lambda) \Theta_{11}^{(k)}(\lambda)
$$

where

$$
\begin{aligned}
& \Theta_{22}^{(k)}(\lambda)=\left(\begin{array}{cc}
V^{(k)} & 0 \\
0 & \Theta_{Z}(\lambda)
\end{array}\right), \\
& \Theta_{11}^{(k)}(\lambda)=\left(\begin{array}{cc}
V_{11}^{(k)} & 0 \\
0 & \Omega_{-}^{(k)}(\lambda)
\end{array}\right), \\
& \Theta_{33}^{(k)}(\lambda)=\left(\begin{array}{cc}
V_{33}^{(k)} & 0 \\
0 & \Omega_{+}^{(k)}(\lambda)
\end{array}\right)
\end{aligned}
$$

and $V_{i i}^{(k)}, i=1,2,3$ are unitary operators. Now $Z_{11}$ and $Z_{33}$ are very simple operators and we can determine $\Omega_{ \pm}^{(k)}(\lambda)$ explicitly. For any contraction $T$ we have

$$
\Theta_{T}(\lambda)=\left.\left[-T+\sum_{n=1}^{\infty} \lambda^{n} D_{T^{*}} T^{* n-1} D_{T}\right]\right|_{D_{T},}, \quad|\lambda|<1
$$

Recalling that $Z_{11}$ and $Z_{33}$ are right shifts, we can apply (3.6) to $Z_{11}$ and $Z_{33}$ to obtain

$$
\Omega_{ \pm}^{(k)}(\lambda)=\lambda^{k} V_{ \pm}^{(k)}, \quad k=0,1,2, \ldots
$$

for some unitary operators $V_{ \pm}^{(k)}$. Thus after successively substituting (3.7) into 
(3.5) and (3.5) into (3.4) we obtain the lemma.

We note that by Lemma 3.1, for $\lambda \neq 0, \Theta_{Z(k)}(\lambda)$ is invertible if and only if $\Theta_{Z}(\lambda)$ is invertible. At $\lambda=0$ we see by (3.4) and (3.5) that $\Theta_{Z(k)}(\lambda)$ has a zero of order at least $2 k$. Thus except for $\lambda=0$ we can use $\Theta_{Z(k)}(\lambda)$ to study the zeroes of $\Theta_{z}(\lambda)$.

The next lemma enables us to avoid this problem at $\lambda=0$.

We say an operator $T$ on $H$ belongs to $C_{0}$. (i.e. $T \in C_{0}$ ) if $T^{n} x \rightarrow 0$ for all $x \in H$. Similarly we say $T \in C_{.0}$ if $T^{* n} x \rightarrow 0$ for all $x \in H$. Now let

$$
\begin{aligned}
T_{a} & =(T-a I)(I-\bar{a} T)^{-1}, \quad|a|<1, \\
& =(T-a I) \sum_{j=0}^{\infty}(\bar{a} T)^{j} .
\end{aligned}
$$

Any invariant subspace $D$ of $T$ is clearly invariant for $T_{a}$. Since $\left(T_{a}\right)_{-a}=T$ the converse is true. Thus $T$ and $T_{a}$ have the same invariant subspaces.

Let $D$ be invariant for $T$. Then $T$ has the form $\left(\begin{array}{ll}A & X \\ 0 & B\end{array}\right)$ where $A: D \rightarrow D$; $B: D \rightarrow D ; X: D \rightarrow D$. Clearly

$$
\begin{aligned}
T_{a} & =(T-a I)(I-\bar{a} T)^{-1} \\
& =\left(\begin{array}{cc}
(A-a I) & X \\
0 & B-a I
\end{array}\right)\left(\begin{array}{cc}
(I-\bar{a} A) & -\bar{a} X \\
0 & (I-\bar{a} B)
\end{array}\right) \\
& =\left(\begin{array}{cc}
(A-a I) & X \\
0 & (B-a I)
\end{array}\right)\left(\begin{array}{cc}
(I-\bar{a} A)^{-1} & (I-\bar{a} A)^{-1}(\bar{a} X)(I-\bar{a} B)^{-1} \\
0 & (I-\bar{a} B)^{-1}
\end{array}\right) \\
& =\left(\begin{array}{cc}
A_{a} & Y \\
0 & B_{a}
\end{array}\right)
\end{aligned}
$$

where

$$
Y=A_{a} \bar{a} X(I-\bar{a} B)^{-1}+X(I-\bar{a} B)^{-1}=\left(\bar{a} A_{a}+I\right) X(I-\bar{a} B)^{-1} .
$$

Thus by (3.8) and (3.9) we see

$$
\left.T_{a}\right|_{D}=A_{a}=\left(\left.T\right|_{D}\right)_{a}
$$

Now if $T_{D}$ is an isometry we know (see $\S I .4 .4$ of [9]) that $\left(\left.T\right|_{D}\right)_{a}$ is an isometry. Thus by (3.10) we see $\left.T_{a}\right|_{D}$ is an isometry. Suppose in addition that $\left.T\right|_{D}$ is completely nonunitary. Then $\left.T\right|_{D} \in C_{.0}$ (i.e. $\left(\left.T\right|_{D}\right)^{* n} \rightarrow 0$ as $\left.n \rightarrow \infty\right)$. By Proposition 2.20 we see that this is equivalent to $\Theta_{\left.T\right|_{D}}(\lambda)$ being inner. But then $\Theta_{\left.T\right|_{D}}((\lambda-a) /(1-\bar{a} \lambda))$ is also inner. By $\S \mathrm{VI} .1 .3$ of [9] we see that this implies $\Theta_{\left(\left.T\right|_{D}\right)_{a}}(\lambda)$ is inner or equivalently that $\left(\left.T\right|_{D}\right)_{a} \in C_{.0}$. By (3.10) we see $\left.T_{a}\right|_{D} \in$ 
$C_{.0}$ and is therefore completely nonunitary.

Finally assume $Y=\left.P_{D^{1}} T\right|_{D^{1}} \in C_{0}$. (i.e. positive powers of the operator converge strongly to zero). Again by Proposition 2.20 we see that $\Theta_{Y}(\lambda)$ is *inner. Thus $\Theta_{Y}((\lambda-a) /(1-\bar{a} \lambda))=\Theta_{Y_{a}}(\lambda)$ is *-inner and we have (by the same proposition) $\left(\left.P_{D^{1}} T\right|_{D^{1}}\right)_{a} \in C_{0}$. Now by (3.9) we see

$$
\left.P_{D^{1}} T_{a}\right|_{D^{\perp}}=B_{a}=\left(\left.P_{D^{1}} T\right|_{D^{1}}\right)_{a} \text {. }
$$

Thus we conclude $\left.P_{D^{1}} T_{a}\right|_{D^{1}} \in C_{0}$.

By these calculations we see that $T$ and $T_{a}$ both satisfy (1.22)-(1.25). We note that (1.24) is satisfied since we have shown $\left.T_{a}\right|_{D}$ is an isometry and $\left.T\right|_{D} \in$ $C_{.0}$. We conclude from the Wold decomposition (see Theorem I.11 of [9]) that $\left.T_{a}\right|_{D}$ is unilateral shift. From this it follows easily that (1.24) holds. By Proposition I.4.3 of [9] we see that $U_{a}$ is the minimal unitary dilation of $T_{a}$. Since $U_{a}$ is also the minimal unitary dilation of $P_{E_{0}}^{H}(k) T_{a} P_{E_{0}}^{H}(k)$ (see Proposition 2.9) we can apply Proposition 1.4.3 of [9] to conclude that

$$
Z(k)_{a}=P_{E_{0}(k)} T_{a} P_{E_{0}}^{H}(k) \text {. }
$$

Similarly $\left.U_{a}\right|_{M\left(O_{-}\right) \vee M\left(O_{+}\right)}$is the minimal unitary dilation of $\left.P_{J(k)} U_{a}\right|_{J(k)}$, and we can conclude from Proposition 1.4.3 of [9] that $\Sigma(k)_{a}=P_{J(k)} U_{a} i_{J(k)}$. Thus all theorems previously proven about the $T, \Sigma(k), Z(k)$ system apply equally well to the $T_{a}, \Sigma(k)_{a}, Z(k)_{a}$ system. We record this as

LEMMA 3.2. The discrete framework generated by $T$ on $H$ is equivalent to the discrete framework generated by $T_{a}$ on $\mathrm{H}$.

In particular we can restate Corollary 2.14 in the $T_{a}$ framework as

$$
\begin{aligned}
\Theta_{Z(k)_{a}}(\lambda)\left[U_{a}-Z(k)_{a}\right] e_{0}(k) \\
\quad=\Theta_{\Sigma(k)_{a}^{*}}(\lambda)\left[T_{a}-Z(k)_{a}\right] e_{0}(k)+\Theta_{T_{a}}(\lambda)\left[U_{a}-T_{a}\right] e_{0}(k) .
\end{aligned}
$$

If we replace $\lambda$ with $\lambda=(\mu-a) /(1-\bar{a} \mu)$, by $\S$ VI.1.3 of [9] we have

$$
\begin{aligned}
\Theta_{Z(k)}(\mu) & {\left[U_{a}-Z(k)_{a}\right] e_{0}(k) } \\
& =\Theta_{\Sigma(k) *} *(\mu)\left[T_{a}-Z(k)_{a}\right] e_{0}(k)+\Theta_{T}(\mu)\left[U_{a}-T_{a}\right] e_{0}(k) .
\end{aligned}
$$

We see that (3.11), a theorem given in terms of the $T_{a}$ system, can be replaced, as in (3.12), by a theorem involving the $T$ system. What is important to note is that the points symmetric with respect to the unit circle remain symmetric after applying the fractional linear transformation $\lambda=(\mu-a) /(1-\bar{a} \mu)$. Thus theorems involving symmetric points and Corollary 2.14 can be stated in either the $T$ or $T_{a}$ framework. We use this fact to advantage later.

We next prove discrete versions of Theorems 2 and 3 of the Introduction. 
THEOREM 3.3. For $k=2,3, \ldots$ let $\Theta_{z(k)}(\lambda)$ have an analytic extension through (part of) the unit circle to a connected open set $\Gamma$ containing the open unit disc. Then $\widetilde{S}_{\mathrm{red}}^{(k)}(\lambda)$ and $\Theta_{T}(\lambda)$ have analytic extensions to $\Gamma$. Assume $\left|\lambda_{0}\right|$ $<1$ and $1 / \lambda_{0}$ belongs to the closure of $\Gamma^{*}$ where ${ }^{*}$ denotes reflection through the $x$-axis. If $1 / \lambda_{0}$ is a pole or essential singularity of $S_{\mathrm{red}}^{(k)}(\lambda)$ then $\lambda_{0}$ is not a point where $\Theta_{z(k)}(\lambda)$ is an isomorphism.

Proof. By assumption $\Theta_{Z(k)}(\lambda)$ has an analytic extension to $\Gamma$. We denote the extension by the same symbol. By Corollary 2.14 it is clear that both $\Theta_{T}(\lambda)$ and $\widetilde{S}_{\text {red }}^{(k)}(\lambda)$ can be extended analytically to $\Gamma$. Again we use the same symbol to denote these extensions. Clearly $S_{\text {red }}^{(k)}(\lambda)$ has an analytic extension to $\Gamma^{*}$. Since $1 / \lambda_{0}$ belongs to the closure of $\Gamma^{*}$ and is a pole or essential singularity of $S_{\mathrm{red}}^{(k)}(\lambda)$, we conclude $1 / \bar{\lambda}_{0}$ belongs to the closure of $\Gamma$ and is a pole or essential of $\widetilde{S}_{\text {red }}^{(k)}(\lambda)$. From Corollary 2.14 we see that $1 / \lambda_{0}$ is a pole or essential singularity of $\Theta_{Z(k)}(\lambda)$. Since $\Theta_{Z(k)}(\lambda)$ is inner and *-inner (see Corollary 2.21), it is unitary almost everywhere on the unit circle. By appealing to the Schwarz reflection principle for operator-valued functions (see Chapter III of [6]) we see $\lambda_{0}$ is not a point where $\Theta_{z(k)}(\lambda)$ is an isomorphism.

THEOREM 3.4. For $k=2,3, \ldots$ let $\Theta_{Z(k)}(\lambda)$ have an analytic extension through (part of) the unit circle to an open connected set $\Gamma$ containing the open unit disc. Assume $|\lambda|_{0}<1$ and $1 / \bar{\lambda}_{0} \in \Gamma$. Then $1 / \lambda_{0}$ is a point where $S_{\text {red }}^{(k)}(\lambda)$ is injective if and only if $\lambda_{0}$ is a point where $\theta_{T}(\lambda)$ is injective.

Proof. The proof proceeds by a (finite) sequence of claims.

Claim 1. Let $\phi: E_{0}(k) \rightarrow E_{0}(k)$ be defined by

$$
\phi\left(\lambda_{0}\right) e_{0}(k)=\left(I-\lambda_{0} Z(k)^{*}\right)^{-1}\left(\lambda_{0}-Z(k)\right) e_{0}(k)
$$

Then $\phi\left(\lambda_{0}\right)$ is an isomorphism.

PROOF. By Corollary 2.21 we can apply the Schwarz reflection principle to $\Theta_{z(k)}(\lambda)$. Since $1 / \bar{\lambda}_{0} \in \Gamma$ we conclude

$$
\Theta_{Z(k)}\left(\lambda_{0}\right)=\left[\Theta_{z(k)}\left(1 / \lambda_{0}\right)^{*}\right]^{-1}
$$

The mapping inside the square brackets is bounded since $1 / \lambda_{0} \in \Gamma$ and has a bounded everywhere defined inverse-namely $\Theta_{Z(k)}\left(\lambda_{0}\right)$. Thus $\Theta_{Z(k)}(\lambda)$ is an isomorphism at $\lambda=\lambda_{0}$. By Proposition 2.4 we conclude that $\lambda_{0}$ belongs to the resolvent set of $Z(k)$. Since $\left|\lambda_{0}\right|<1$ it is clear that $\phi$ defined by (3.14) is an isomorphism.

CLAIM 2. $\Theta_{T}(\lambda)$ is injective at $\lambda=\lambda_{0}$ if and only if

$$
\left.\left(I-\lambda_{0} Z(k)^{*}\right)^{-1}\left(\lambda_{0}-Z(k)\right) \operatorname{ker}(U-T)\right|_{E_{0}(k)}=\left.\operatorname{ker}\left(U^{*}-T^{*}\right)\right|_{E_{0}(k)} .
$$


ProOF. We recall a part of (2.94):

$$
\Theta_{T}\left(\lambda_{0}\right)[U-T] e_{0}(k)=U\left[U^{*}-T^{*}\right]\left[I-\lambda_{0} Z(k)^{*}\right]^{-1}\left[\lambda_{0}-Z(k)\right] e_{0}(k) .
$$

Since $\Theta_{T}\left(\lambda_{0}\right) 0=0$ we always have the inclusion

$$
\left.\left.\left(I-\lambda_{0} Z(k)^{*}\right)^{-1}\left(\lambda_{0}-Z(k)\right) \operatorname{ker}(U-T)\right|_{E_{0}(k)} \subset \operatorname{ker}\left(U^{*}-T^{*}\right)\right|_{E_{0}(k)}
$$

Using (3.14) we can rewrite (3.17) and (3.18) respectively as

$$
\Theta_{T}\left(\lambda_{0}\right)[U-T] e_{0}(k)=U\left(U^{*}-T^{*}\right) \phi\left(\lambda_{0}\right) e_{0}(k)
$$

and

$$
\left.\left.\phi\left(\lambda_{0}\right) \operatorname{ker}(U-T)\right|_{E_{0}(k)} \subset \operatorname{ker}\left(U^{*}-T^{*}\right)\right|_{E_{0}(k)} .
$$

Since $\phi\left(\lambda_{0}\right)$ is onto by Claim 1, the inclusion in (3.20) is proper if and only if there exists an $e_{0}(k) \in E_{0}(k)$ with the following two properties:

$$
\begin{gathered}
\left.e_{0}(k) \notin \operatorname{ker}(U-T)\right|_{E_{0}(k)}, \\
\left.\phi\left(\lambda_{0}\right) e_{0}(k) \in \operatorname{ker}\left(U^{*}-T^{*}\right)\right|_{E_{0}(k)} .
\end{gathered}
$$

By (3.22) and (3.19) we see $e_{0}(k)$ enjoys properties (3.21) and (3.22) if and only if

$$
\begin{gathered}
\Theta_{T}\left(\lambda_{0}\right)[U-T] e_{0}(k)=0, \\
{[U-T] e_{0}(k) \neq 0}
\end{gathered}
$$

i.e. if and only if $\Theta_{T}\left(\lambda_{0}\right)$ is not injective. This concludes the proof of Claim 2 .

Claim 3. $S_{\text {red }}^{(k)}(\lambda)$ is injective at $\lambda=1 / \lambda_{0}$ if and only if

$$
\left(I-\lambda_{0} Z(k)^{*}\right)^{-1}\left(\lambda_{0}-Z(k)\right) \operatorname{ker}(T-Z(k))=\operatorname{ker}\left(T^{*}-Z(k)^{*}\right) .
$$

Proof. We recall a part of (2.99):

$$
S_{\text {red }}^{(k)}(\lambda)\left[T^{*}-Z(k)^{*}\right] e_{0}(k)=U^{*}(T-Z(k))(I-\lambda Z(k))^{-1}\left(\lambda-Z(k)^{*}\right) e_{0}(k) .
$$

Since $S_{\text {red }}^{(k)}(\lambda) 0=0$ we always have the inclusion

$$
\left.\left.(I-\lambda Z(k))^{-1}\left(\lambda-Z(k)^{*}\right) \operatorname{ker}\left(T^{*}-Z(k)^{*}\right)\right|_{E_{0}(k)} \subset \operatorname{ker}(T-Z(k))\right|_{E_{0}(k)}
$$

Letting $\lambda=1 / \lambda_{0}$ and applying (3.14) we rewrite (3.26) and (3.27) as

$$
\begin{gathered}
S_{\mathrm{red}}^{(k)}\left(1 / \lambda_{0}\right)\left[T^{*}-Z(k)^{*}\right] e_{0}(k)=U^{*}(T-Z(k)) \phi^{-1}\left(\lambda_{0}\right) e_{0}(k), \\
\left.\left.\phi\left(\lambda_{0}\right)^{-1} \operatorname{ker}\left(T^{*}-Z(k)^{*}\right)\right|_{E_{0}(k)} \subset \operatorname{ker}(T-Z(k))\right|_{E_{0}(k)} .
\end{gathered}
$$

Since $\phi^{-1}\left(\lambda_{0}\right)$ is onto by Claim 1 the inclusion in (3.29) is proper if and only if 
there exists an $e_{0}(k) \in E_{0}(k)$ with the following two properties

$$
\begin{gathered}
\left.e_{0}(k) \notin \operatorname{ker}\left(T^{*}-Z(k)^{*}\right)\right|_{E_{0}(k)}, \\
\left.\phi^{-1}\left(\lambda_{0}\right) e_{0}(k) \in \operatorname{ker}(T-Z(k))\right|_{E_{0}(k)} .
\end{gathered}
$$

Applying (3.31) to (3.28) we see $e_{0}(k)$ enjoys properties (3.30) and (3.31) if and only if

$$
\begin{gathered}
S_{\text {red }}^{(k)}\left(1 / \lambda_{0}\right)\left[T^{*}-Z(k)^{*}\right] e_{0}(k)=0, \\
{\left[T^{*}-Z(k)^{*}\right] e_{0}(k) \neq 0}
\end{gathered}
$$

i.e. if and only if $S_{\text {red }}^{(k)}\left(1 / \lambda_{0}\right)$ is not injective.

Claim 4. $\Theta_{Z(k) *}(\lambda)$ is injective at $\lambda=1 / \lambda_{0}$ if and only if

$$
\begin{gathered}
\left.\left(I-\lambda_{0} Z(k)\right)^{-1}\left(\lambda_{0}-Z(k)^{*}\right) \operatorname{ker}\left(U^{*}-Z(k)^{*}\right)\right|_{E_{0}(k)} \\
=\left.\operatorname{ker}(U-Z(k))\right|_{E_{0}(k)} .
\end{gathered}
$$

Proof. We recall a part of (2.99):

$$
\begin{aligned}
\Theta_{Z(k) *}(\lambda)\left[U^{*}-Z(k)^{*}\right] e_{0}(k) \\
=U^{*}(U-Z(k))(I-\lambda Z(k))^{-1}\left(\lambda-Z(k)^{*}\right) e_{0}(k) .
\end{aligned}
$$

Since $\Theta_{Z(k)} *(\lambda) 0=0$ we always have the inclusion

$$
\left.\left.(I-\lambda Z(k))^{-1}\left(\lambda-Z(k)^{*}\right) \operatorname{ker}\left(U^{*}-Z(k)^{*}\right)\right|_{E_{0}(k)} \subset \operatorname{ker}(U-Z(k))\right|_{E_{0}(k)}
$$

Letting $\lambda=1 / \lambda_{0}$ we can rewrite (3.35) and (3.36) as

$$
\begin{gathered}
\Theta_{Z(k) *}\left(1 / \lambda_{0}\right)\left[U^{*}-Z(k)^{*}\right] e_{0}(k)=U(U-Z(k)) \phi^{-1}\left(\lambda_{0}\right) e_{0}(k), \\
\left.\left.\phi^{-1}\left(\lambda_{0}\right) \operatorname{ker}\left(U^{*}-Z(k)^{*}\right)\right|_{E_{0}(k)} \subset \operatorname{ker}(U-Z(k))\right|_{E_{0}(k)}
\end{gathered}
$$

Since $\phi^{-1}$ is onto by Claim 1 the inclusion in (3.38) is proper if and only if there exists an $e_{0}(k) \in E_{0}(k)$ with the following two properties:

$$
\begin{gathered}
\left.e_{0}(k) \notin \operatorname{ker}\left(U^{*}-Z(k)^{*}\right)\right|_{E_{0}(k)}, \\
\left.\phi^{-1}\left(\lambda_{0}\right) e_{0}(k) \in \operatorname{ker}(U-Z(k))\right|_{E_{0}(k)} .
\end{gathered}
$$

Applying (3.40) to (3.35) we see $e_{0}(k)$ enjoys properties (3.39) and (3.40) if and only if

$$
\begin{gathered}
\Theta_{Z(k) *}\left(1 / \lambda_{0}\right)\left[U^{*}-Z(k)^{*}\right] e_{0}(k)=0 \\
{\left[U^{*}-Z(k)^{*}\right] e_{0}(k) \neq 0}
\end{gathered}
$$


i.e. if and only if $\Theta_{Z(k) *}\left(1 / \lambda_{0}\right)$ is not injective.

Claim 5. If $1 / \bar{\lambda}_{0} \in \Gamma$, then $\Theta_{Z(k)} *(\lambda)$ is injective at $\lambda=1 / \lambda_{0}$.

Proof. Assume $1 / \bar{\lambda}_{0} \in \Gamma$. By Corollary 2.21 and the Schwarz reflection principle we can write

$$
\Theta_{z(k)}\left(\lambda_{0}\right)^{*}=\left[\Theta_{z(k)}\left(1 / \bar{\lambda}_{0}\right)\right]^{-1} .
$$

The mapping inside the square brackets is bounded since $1 / \lambda_{0} \in \Gamma$ and has a bounded everywhere defined inverse-namely $\Theta_{z(k)}\left(\lambda_{0}\right)^{*}$. Thus $\Theta_{Z(k)}(\lambda)$ is an isomorphism at $\lambda=1 / \bar{\lambda}_{0}$. By (2.8) applied to $Z(k)$ we have

$$
\Theta_{Z(k) *}\left(1 / \lambda_{0}\right)=\Theta_{Z(k)}\left(1 \bar{\lambda}_{0}\right)^{*}
$$

Since the adjoint of an isomorphism is injective the result follows immediately.

We are now ready to prove the only if part of the theorem. We assume $1 / \bar{\lambda}_{0} \in \Gamma$. By Claims 5 and 4 we conclude (3.34), i.e.,

$$
\phi\left(\left.\operatorname{ker}(U-Z(k))\right|_{E_{0}(k)}\right)=\left.\operatorname{ker}\left(U^{*}-Z(k)^{*}\right)\right|_{E_{0}(k)} .
$$

Since we are assuming that $\Theta_{T}\left(\lambda_{0}\right)$ is injective we can also apply Claim 2 to conclude (3.16), i.e.,

$$
\phi\left(\left.\operatorname{ker}(U-T)\right|_{E_{0}(k)}\right)=\left.\operatorname{ker}\left(U^{*}-T^{*}\right)\right|_{E_{0}(k)} .
$$

We note that

$$
\left.\operatorname{ker}(U-Z(k))\right|_{E_{0}(k)}=\left.\left.\operatorname{ker}(U-T)\right|_{E_{0}(k)} \cap \operatorname{ker}(T-Z(k))\right|_{E_{0}(k)}
$$

and

(3.47) $\left.\operatorname{ker}\left(U^{*}-Z(k)^{*}\right)\right|_{E_{0}(k)}=\left.\left.\operatorname{ker}\left(U^{*}-T^{*}\right)\right|_{E_{0}(k)} \cap \operatorname{ker}\left(T^{*}-Z(k)^{*}\right)\right|_{E_{0}(k)}$

Since $\phi$ is an isomorphism it is clear from (3.44) and (3.45) that

(3.48) $\phi[\operatorname{ker}(U-T) \backslash \operatorname{ker}(U-Z(k))]=\left[\operatorname{ker}\left(U^{*}-T^{*}\right) \operatorname{ker}\left(U^{*}-Z(k)^{*}\right)\right]$.

In (3.48) "V" denotes set complementation; we have omitted the symbols " $E_{E_{0}}(k)$ " and will continue to do so for the rest of this proof. To prove the only if part of the theorem it suffices, by Claim 3, to show

$$
\phi\left(\operatorname{ker}(T-Z(k))=\operatorname{ker}\left(T^{*}-Z(k)^{*}\right) .\right.
$$

To this end we note that $T=U$ on $E_{ \pm}(k)$ and $Z(k)=T$ on $E_{0}(k)$ for $k=2,3$, .... We conclude that

$$
\begin{aligned}
& E_{0}(k)=\operatorname{ker}(U-T)+\operatorname{ker}(T-Z(k)), \\
& E_{0}(k)=\operatorname{ker}\left(U^{*}-T^{*}\right)+\operatorname{ker}\left(T^{*}-Z(k)^{*}\right) .
\end{aligned}
$$

Thus by (3.46) and (3.47) we can decompose and $e_{0}(k) \in E_{0}(k)$ as $e_{0}(k)=x$ 
$+y$ where $x \in \operatorname{ker}(U-T) \operatorname{ker}(U-Z(k)), y \in \operatorname{ker}(T-Z(k))$. This decomposition is not unique. Nevertheless applying $\phi$ to $x$ we see by (3.48) that $\phi x \in$ $\operatorname{ker}\left(U^{*}-T^{*}\right) \operatorname{ker}\left(T^{*}-Z(k)^{*}\right)$. Since $\phi$ is an isomorphism $\phi x \neq 0$ unless $x=$ 0 . Thus by (3.46), (3.47), and (3.50) we conclude that for any $e_{0}(k) \in E_{0}(k)$, $\phi e_{0}(k) \in \operatorname{ker}\left(T^{*}-Z(k)^{*}\right)$ if and only if $x=0$, i.e., if and only if $e_{0}(k) \in$ $\operatorname{ker}(T-Z(k))$. Thus since $\phi$ maps onto $E_{0}(k)$ we conclude (3.49). The converse is proven similarly.

THEOREM 3.5. For $k=2,3, \ldots$ let $\Theta_{Z(k)}(\lambda)$ have an analytic extension through (part of) the unit circle to a connected open set $\Gamma$ containing the open unit disc. Let $\left|\lambda_{0}\right|<1$ and $1 / \lambda_{0}$ belong to the closure of $\Gamma^{*}$. Finally assume $T$ has no eigenvectors which are contained in $E_{0}\left(=E_{0}(0)\right)$. Then if $\lambda_{0}$ is a zero of $\Theta_{Z(k)}(\lambda), 1 / \lambda_{0}$ is a pole or essential singularity of $S_{\mathrm{red}}^{(k)}(\lambda)$.

Proof. If $\lambda_{0}$ is a zero of $\Theta_{Z(k)}(\lambda)$, then by Proposition 2.4 we see $\lambda_{0}$ $\in \sigma_{p}(Z(k))$. Since $\left|\lambda_{0}\right|<1$ we know $\left[1 / \lambda_{0}-Z(k)\right]^{-1}$ is an isomorphism and for $\mu$ near $\lambda_{0},[1 / \mu-Z(k)]^{-1}$ is uniformly bounded by some constant $M$. Let $f$ be a unit eigenvector associated with $\lambda_{0} \in \sigma_{p}(Z(k))$. Then $(\mu-Z(k))^{-1} f=$ $f /\left(\mu-\lambda_{0}\right)$. From (2.99) we have

$$
S_{\mathrm{red}}^{(k)}(1 / \mu)\left[T^{*}-Z(k)^{*}\right] e_{0}(k)=U^{*}(T-Z(k))(\mu-Z(k))^{-1}\left(I-\mu Z(k)^{*}\right) e_{0}(k)
$$

and so

$$
S_{\mathrm{red}}^{(k)}(1 / \mu)\left[T^{*}-Z(k)^{*}\right]\left[\left(I-\mu Z(k)^{*}\right)^{-1} f\right]=\frac{\mu}{M\left(\mu-\lambda_{0}\right)} U^{*}(T-Z(k)) f .
$$

Thus to show $1 / \lambda_{0}$ is a pole or essential singularity of $S_{\text {red }}^{(k)}(\lambda)$ we need only show that $(T-Z(k))$ does not kill the eigenspace of $Z(k)$ at $\lambda_{0}$. Let $E_{0}(k)=$ $F_{+}(k) \oplus E_{0} \oplus F_{-}(k)$ where the notation is as in (3.1). Since $f \in E_{0}(k)$ and $\lambda_{0}^{j} f=Z(k)^{j} f$ we see the $F_{-}(k)$ part of $f$ is zero.

Now assume that $(T-Z(k))$ kills the eigeispace of $Z(k)$ at $\lambda_{0}$. Then $(T-Z(k)) f=0$, i.e., $T f=Z(k) f=\lambda_{0} f$. Let $(g)_{j}$ denote the $O_{+}(j)$ component of g. Then we have for $j \in \mathbf{Z}_{+}$

$$
(f)_{j}=(T f)_{j+1}=(Z(k) f)_{j+1}=\lambda_{0}(f)_{j+1} .
$$

Since $0<\left|\lambda_{0}\right|<1$ we see that either $\left\{(f)_{j}\right\}_{j=0}^{\infty} \notin l^{2}$, or $(f)_{j}=0$ for all $j$. If the latter occurs we see $f \in E_{0}$ and this contradicts the fact that $T$ has no eigenvectors in $E_{0}$. Thus $(T-Z(k))$ does not kill $f$, and the theorem is proven.

We conclude this section with

THEOREM 3.6. If $\Theta_{Z}(\lambda)$ is inner and *inner, $\Theta_{Z}(\lambda)$ has an analytic extension through an arc $(\gamma)$ contained in the unit circle, if and only if $\sigma(z) \cap(\gamma)=\varnothing$.

Proof. By assumption $\Theta_{z}\left(e^{i t}\right)$ is unitary almost everywhere on the unit 
circle. If $\Theta_{Z}(\lambda)$ has an analytic extension through $(\gamma)$ it is clearly unitary for all $e^{i t} \in(\gamma)$. But then by Proposition 2.4 we see $\sigma(z) \cap(\gamma)=\varnothing$.

Conversely if $\sigma(Z) \cap(\gamma)=\varnothing$ we see by the same theorem that $\Theta_{Z}\left(e^{i t}\right)$ is unitary for all $e^{i t} \in(\gamma)$, and that $\Theta_{Z}(\lambda)$ is boundedly invertible for $|\lambda| \leqslant 1$ and sufficiently close to compact subsets of $(\gamma)$. Now $\left\|\Theta_{Z}(\lambda)^{-1}\right\|$ is a continuous function on this compact set and is thus uniformly bounded. By Lemma III.1.3 of [6] we see $\Theta_{Z}(\lambda)$ can be extended through $(\gamma)$.

4. Applications to Lax-Phillips scattering theory. In this section we find continuous versions of Theorems 3.3-3.5. We then show how these results apply to some systems considered by Lax and Phillips. In particular we show $\S \S 1-3$ of this paper provide an abstract framework for the systems considered in [7] and [8].

We now translate Theorems 3.3-3.5 into a continuous framework. Consider the fractional linear transformation

$$
T: \lambda \mapsto z=(\lambda+1) /(\lambda-1)
$$

which maps the unit disc onto the left half plane. For any contraction $T$ and its Cayley transform $A$ define

$$
\Theta_{A}(z)=\Theta_{T}(\lambda)
$$

whenever $\lambda$ belongs to the domain of analyticity of $\Theta_{T}$. Since $\Theta_{T}(\lambda)$ is analytic in the disc, $\Theta_{A}(z)$ is analytic in the left half plane. For any operator-valued function $\gamma\left(e^{i t}\right)$ defined on the circle we can define $\gamma(\sigma)$ on the imaginary axis by setting $\gamma(\sigma)=\gamma\left(e^{i t}\right)$. Let

$$
\Theta_{A}^{\sim}(z)=\Theta_{A}(z)^{*}
$$

Then by (4.2) it is easy to see that

$$
\Theta_{A} *(z)=\Theta_{A}^{\sim}(z) \text {. }
$$

By Theorem 2.4 if $\left|\lambda_{0}\right|<1$ then $\lambda_{0} \in \sigma(T)$ if and only if $\Theta_{T}\left(\lambda_{0}\right)$ is not an isomorphism. Since $A=(T+I)(T-I)^{-1}$ we see by the spectral mapping theorem and (4.1) that if $z_{0}=\tau\left(\lambda_{0}\right)$ then $z_{0} \in \sigma(A)$ if and only if $\Theta_{T}\left(\lambda_{0}\right)$ is not an isomorphism. Let $\Delta=\tau \Gamma=\{\tau(\lambda) \mid \lambda \in \Gamma\}$. Then clearly $\Theta_{T}(\lambda)$ has an analytic extension to $\Gamma$ if and only if $\Theta_{A}(z)$ has an analytic extension to $\Delta$.

Let $A, B(k)$, and $C(k)$ denote the Cayley transforms of $T, Z(k)$, and $\Sigma(k)$ respectively. If we recall (see Corollary 2.7$)$ that $\widetilde{S}_{\text {red }}^{(k)}(\lambda)=\Theta_{\Sigma(k)}^{\sim}(\lambda)$ we can apply these ideas to all three characteristic functions in Theorems 3.3-3.5.

For all of these theorems the case $z_{0}=-1\left(\lambda_{0}=0\right)$ presents special problems. Because $\Theta_{B(k)}(z)$ has a zero at $z_{0}=-1$ for $k=1,2, \ldots$ (recall (3.5) 
and (4.1)) we see by Corollary 2.21 and the Schwarz reflection principle that 1 $\notin \Delta$. Thus the point 1 never satisfies the hypotheses of these theorems.

To avoid this "blindspot" we proceed as follows. Consider the discrete framework and denote $Z(0)$ by $Z, S_{\text {red }}^{(0)}(\lambda)$ by $S_{\text {red }}(\lambda)$, etc. For the moment assume zero is a point of isomorphism for $\Theta_{Z}(\lambda), S_{\text {red }}(\lambda)$, and $\Theta_{T}(\lambda)$. We will show later that this assumption can be replaced by a much weaker assumption.

By (3.4) we see that except for $\lambda=0, \Theta_{Z}(\lambda)$ and $\Theta_{Z(k)}(\lambda), k=1,2, \ldots$, are isomorphic at exactly the same points of the complex plane. Similarly by Corollary 2.2 we see that, except for $\lambda=0, S_{\text {red }}(\lambda)$ and $S_{\text {red }}^{(k)}(\lambda), k=1,2, \ldots$, are isomorphic at exactly the same points of the complex plane. Thus we can replace any statement in Theorems 3.3-3.5 concerning the nonisomorphic points of $\Theta_{Z(k)}(\lambda)$ and $S_{\text {red }}^{(k)}(\lambda), k=2,3, \ldots$, by the same statements with $\Theta_{Z(k)}(\lambda)$ replaced by $\Theta_{Z}(\lambda)$ and $S_{\text {red }}^{(k)}(\lambda)$ replaced by $S_{\text {red }}(\lambda)$. The "problem" at zero disappears since we have assumed that all the operator-valued analytic functions are isomorphisms there. As before we can now translate these theorems into the continuous framework (i.e. the left half plane). When we do this the special problems with $z_{0}=-1$ disappear.

Before stating the resulting theorems we show that it is alway possible to assume $\lambda=0$ is a point of isomorphism for $\Theta_{Z}(\lambda), S_{\text {red }}(\lambda)$, and $\Theta_{T}(\lambda)$-at least for all systems which satisfy a mild regularity condition. We assume that we can find an $a$ with $-1<a<1$ such that $a \notin \sigma(T), a \notin \sigma(Z)$, and $\Theta_{\Sigma(k)}(a)$ is an isomorphism. In all the systems considered by Lax and Phillips this condition is certainly satisfied. We come back to this point later. From now on we make the standing assumption that this condition is satisfied.

Now consider the discrete framework involving $T_{a}$. By Lemma 3.2 we see the $T_{a}$ system satisfies the same properties as the discrete $T$ system. The one important difference is that zero is now a point of isomorphism for $\Theta_{z_{a}}(\lambda), \Theta_{T_{a}}(\lambda)$, and $S_{\mathrm{red}, a}(\lambda)$. To see this we use Proposition 2.4 and the spectral mapping theorem. We can thus apply our previous discussion to the $T_{a}$ system instead of the $T$ system. We conclude, by recalling the remark after Lemma 3.2, that the special problems involving $z_{0}=-1$ can be avoided.

Some final notes are in order. First by Theorem $3.6 \Delta$ can be explicitly computed once $\sigma(B)$ is known. Since any analytic extension of $\Theta_{B}(z)$ is given by the Schwarz reflection principle we can apply Proposition 2.4 and conclude that $\Delta$ is the component of

$$
\{z=x+i y \mid x-i y \in \rho(B)\} \cup\{z \mid \operatorname{Re} z<0\}
$$

which contains the left half plane. In the applications considered by Lax and Phillips the spectrum of $B$ is discrete and contained in the open left half plane. Thus $\mathrm{C} \triangle \mathrm{is}$ discrete. 
Second Lax and Phillips choose the lower half plane as the half plane where $S(z)$ is analytic. This differs from our choice by an angle of $\pi / 2$. We now restate Theorems 3.3-3.5 with the preceeding remarks in mind.

THEOREM 4.1. $S_{\text {red }}^{\text {cts }}(z)$ has an analytic extension to all but a discrete set of points in the upper half plane where it may have poles or essential singularities. If $z_{0}$ is a pole or essential singularity of $S_{\mathrm{red}}^{\mathrm{cts}}(z)$, then $i z_{0} \in \sigma(B)$.

THEOREM 4.2. If $z_{0}=x_{0}+i y_{0}, x_{0}<0$, and $-x_{0}+i y_{0} \in \Delta$, then $-i z_{0}$ is a zero of $S_{\mathrm{red}}^{\mathrm{cts}}(z)$ if and only if $z_{0} \in \sigma(A)$.

THEOREM 4.3. Assume $A$ has no eigenvectors which are contained in $E_{0}$. Then if $i z_{0} \in \sigma(B), z_{0}$ is a pole or essential singularity of $S_{\mathrm{red}}^{\mathrm{cts}}(z)$.

We now restate Theorem 4.2 slightly. Clearly $-x_{0}+i y_{0} \in \Delta$ if and only if $x_{0}+i y_{0} \notin \sigma(B)$. By Theorems 4.1 and $4.3 z_{0}=x_{0}+i y_{0} \in \sigma(B)$ if and only if $-i z_{0}$ is a pole or essential singularity of $S_{\text {red }}^{\text {cts }}(z)$. Thus we can restate Theorem 4.2 as

THEOREM 4.4. If $z_{0} \in \sigma(B)$ and $\operatorname{Im} z_{0}<0$, then $-i z_{0}$ is a zero or possibly a pole or essential singularity of $S_{\mathrm{red}}^{\mathrm{cts}}(z)$.

Theorems 4.1, 4.3, and 4.4. are similar to Theorems 1 and 2 of the Introduction.

We now discuss the application of these results to the system considered in [8]. In [8] Lax and Phillips consider the acoustic equation in an exterior domain $G \subset \mathbf{R}^{2 n+1}$

$$
\begin{aligned}
u_{t t} & =\Delta u \quad \text { in } G, \\
\partial_{n} u+\alpha u_{t} & =0, \quad \alpha \geqslant 0 \text { on } \partial G .
\end{aligned}
$$

They embed the problem in a Hilbert space $H$ which consists of all initial data of finite energy in $G$. We define $H, T(t)$, and $D_{ \pm}$as in [8]. $T(t)$ is shown to be isometric on $D_{+}$, and $T(t)^{*}$ is shown to be isometric on $D_{-}$. Following the material in the Appendix of [8], we construct $H_{ \pm} \cdot H_{ \pm}$satisfy (1.1)-(1.3). In [8] Lax and Phillips show that (1.4) and (1.5) are satisfied by $T(t)$. Thus the abstract framework applies to [8]. We note that [8] satisfies one additional very important property, namely (2.18). Thus in $\$ 2$ we can take $D_{ \pm}=E_{ \pm}$. Clearly then $\alpha, \alpha\left(e^{i t}\right), \beta$, and $\beta\left(e^{i t}\right)$, defined by (2.29), (2.33), and (2.34), become identity maps. Thus by $(2.30)$ we see

$$
S_{\text {red }}^{(k)}(\lambda)=S^{(k)}(\lambda), \quad k \in Z_{+},
$$

where $S^{(k)}(\lambda)$ is the Fourier representation of $S$ with respect to $N_{ \pm}(k)$. In order 
to conclude Theorems $4.1,4.3$, and 4.4 we will show that $\mathrm{Cl} \Delta$ (defined by (4.5)) is discrete.

In Chapter 5 of [6] it is shown that $Z(t)(K-B)^{-1}$ is compact for some choices of $t$ and $\kappa$. In [6] and [8] this fact is shown to have the following consequences:

(i) $B$ has only discrete point spectra which lies in the open left half plane,

(ii) $(z-B)^{-1}$ is meromorphic in $\mathbf{C}$.

By (i) we see that $\mathrm{Cl} \Delta$ is discrete. We claim that (ii) implies $S(z)$ has no essential singularites. To see this transform to the discrete framework. Then $(\lambda-Z)^{-1}$ is meromorphic away from $\lambda=1$. Thus by Proposition VI.42 of [9] we see that, for $|\lambda|<1, \Theta_{z}(\lambda)^{-1}$ is meromorphic. Since $\Theta_{Z}(\lambda)$ and $\Theta_{Z(k)}(\lambda)$ are related by (3.4), we see that $\Theta_{Z(k)}(\lambda)^{-1}$ is meromorphic in the open unit disc. Now by assumption $\Theta_{z(k)}(\lambda)$ has an analytic extension through the circle. This is given by the Schwarz reflection principle; i.e.,

$$
\Theta_{Z(k)}(1 / \lambda)=\left[\Theta_{Z(k)}(\lambda)^{-1}\right]^{*}, \quad|\lambda|<1 .
$$

Clearly then $\Theta_{Z(k)}(\lambda)$ is meromorphic on $C$ except possibly at $\lambda=1$. Thus by (2.97) $S_{\text {red }}^{(k)}(\lambda)$ is also meromorphic in the same domain. Now switching to the continuous framework we see $S_{\text {red }}^{\text {cts }}(z)$ is meromorphic in $\mathbf{C}$.

In [8] the spectral points of $A$ in the open left half plane are shown to be discrete point spectra for $A$. Also, in the same paper $S(z)$ is shown to be of the form $I+K(z)$ where $K(z)$ is compact. As mentioned there, this is sufficient to insure that the set of nonisomorphic points of $S(z)$ is isolated. Thus we see, by mapping the left half plane to the unit disc, that the mild regularity conditions which we assumed in order to prove Theorems 4.7, 4.9, and 4.10 are satisfied. Finally in [8], $A$ is shown to have no eigenvectors which lie in $H \ominus\left(D_{-} \oplus D_{+}\right)$.

If we restate Theorems $4.1,4.3$, and 4.4 with the preceding comments in mind we obtain Theorems 1 and 2 of the Introduction.

We now turn our attention to the system considered in [7]. We show that $\S \S 1-3$ of this paper apply to this system also. In [7] Lax and Phillips consider the acoustic equation with a potential $q$ which is zero outside a compact set. They consider

$$
u_{t t}=\Delta u-q u
$$

over a domain $G \subset \mathbf{R}^{3}$ exterior to a bounded obstacle. On the boundary of $G$ $u$ satisfies

$$
\partial_{n} u+\sigma u=0 \text {. }
$$

The functions $\sigma$ and $q$ are taken to be real valued. Using the notation of [7] we can set 


$$
\begin{aligned}
H & =\hat{H}_{1}, & D_{ \pm} & =\hat{D}_{ \pm}^{\prime}, \\
T(t) & =\hat{U}(t), & E_{ \pm} & =\hat{D}_{ \pm}^{\prime \prime} .
\end{aligned}
$$

With these definitions Lax and Phillips prove that $H, T(t), D_{ \pm}$, and $E_{ \pm}$ satisfy the assumptions of $\S 2$. Since $D_{+}$is not orthogonal to $D_{-}$we will be working with $S_{\text {red }}$ instead of $S$ (as was the case for the systems in [8] considered previously); also $\alpha\left(e^{i t}\right)$ and $\beta\left(e^{i t}\right)$ are no longer identity maps. Since Lax and Phillips use notation which differs from the notation that we have used in $\S 2$, we provide the following dictionary:

$$
\begin{aligned}
& S_{\text {red }}=S^{\prime \prime}, \quad S_{\text {red }}^{\text {cts }}(z)=S^{\prime \prime}(z), \\
& \alpha=S_{-}, \quad \alpha(\sigma)=S_{-}(\sigma), \quad \sigma \text { real }, \\
& \beta=S_{+}, \quad \beta(\sigma)=S_{+}(\sigma), \quad \sigma \text { real. }
\end{aligned}
$$

In [7] $E_{+}$and $D_{+}$(resp. $E_{-}$and $D_{-}$) are shown to be outgoing (resp. incoming) for $H$. Thus $M\left(E_{ \pm}\right)=M\left(D_{ \pm}\right)=H$ and thus by (2.29) (since $M\left(E_{ \pm}\right)=M\left(O_{ \pm}\right)$ and $M\left(D_{ \pm}\right)=M\left(N_{ \pm}\right)$) we see $S_{\text {red }}, \alpha$ and $\beta$ are identity maps.

The Fourier representation of a unitary operator must be unitary almost everywhere on the unit circle. Thus $S^{\text {cts }}(\sigma), S_{\text {red }}^{\text {cts }}(\sigma), \alpha(\sigma)$, and $\beta(\sigma)$ are unitary almost everywhere on the real axis.

By applying Claim 2.5 and then Cayley transforming we see that $S_{\text {red }}^{\text {cts }}(\sigma)$, $\alpha(\sigma)$, and $\beta(\sigma)$ all have analytic extension to the lower half plane.

All three operators are actually meromorphic in the entire plane. To show that $S_{\text {red }}^{\text {cts }}(z)$ is meromorphic in $\mathbf{C}$ define $Z(t)$ as in (2.55). In [7] $Z(2 \rho)(\kappa-B)^{-1}$ is shown to be compact. We then argue as we did previously for [8] to conclude that Theorem 1 of the Introduction holds for this system also. We note that Theorem 2 of the Introduction is trivial since $A$ is skew selfadjoint and thus has no spectrum in the open left half plane.

To show that $\alpha(z)$ is meromorphic in the complex plane, we first show that it is, after transformation onto the unit disc, a characteristic function of an operator $Z$ defined on $D_{-} \Theta E_{-}$by

$$
Z=P_{H \ominus D_{-}^{\perp}}^{H} U P_{H \ominus E_{-}}^{H} .
$$

Since $D_{-}^{\perp}$ and $E_{-}$are orthogonal this is a semigroup. To show that $\Theta_{Z}=\alpha$ we apply Theorem 2.6 after noting that $D_{-}^{\perp}$ and $E_{-}$have the necessary properties to replace the $E_{+}$and $E_{-}$of the theorem while $\alpha$, given by (2.29), can replace the $S_{\text {red }}$ of the theorem. With these replacements the conclusion of the theorem is precisely that $\alpha(\lambda),|\lambda|<1$, is the characteristic function of $Z$ given by (4.12).

Lemma 2.4 of [7] shows that $D_{-} \ominus E_{-}$is finite dimensional. Thus by 
Proposition 2.4 and the Schwarz reflection principle we conclude that $\alpha(z)$ is meromorphic in the complex plane with only a finite number of zeroes and poles. Since

$$
S(z)=\beta^{-1}(z) S_{\mathrm{red}}(z) \alpha^{-1}(z)
$$

we see that $S(z)$ is meromorphic in the entire plane with only a finite number of poles below the real axis.

\section{REFERENCES}

1. V. M. Adamjan and D. Z. Arov, A class of scattering operators and characteristic operator-functions of contractions, Dokl. Akad. Nauk SSSR 160 (1965), 9-12 = Soviet Math. Dokl. 6 (1965), 1-5. MR 30 \#5169.

2. - Scattering operators and contraction semigroups in Hilbert space, Dokl. Akad. Nauk SSSR 165 (1965), 9-12 = Soviet Math. Dokl. 6 (1965), 1377-1380. MR 32 \#6240.

3. - On unitary couplings of semiunitary operators, Mat. Issled. 1 (1966), no. 2, 3-64; English transl., Amer. Math. Soc. Transl. (2) 95 (1970), 75-129. MR 34 \#6528; 42 \#2899.

4. R. G. Douglas and J. W. Helton, Inner dilations of analytic matrix functions and Darlington synthesis, Acta Sci. Math. (Szeged) 34 (1973), 61-67. MR 48 \#900.

5. C. Foias, On the Lax-Phillips non conservative scattering theory, J. Functional Analysis 19 (1975), 273-301.

6. P. D. Lax and R. S. Phillips, Scattering theory, Pure and Appl. Math., vol. 26, Academic Press, New York, 1967. MR 36 \#530.

7. The acoustic equation with an indefinite energy form and the Schrödinger equation, J. Functional Analysis 1 (1967), 37-83. MR 36 \#531.

8. Scattering theory for dissipative hyperbolic systems, J. Functional Analysis 14 (1973), 172-235.

9. B. Sz.-Nagy and C. Foias, Analyse harmonique des opérateurs de l'espace de Hilbert, Masson, Paris; Akad. Kiadó, Budapest, 1967; English rev. transl., North-Holland, Amsterdam; American Elsevier, New York; Akad. Kiadó, Budapest, 1970. MR 37 \#78; 43 \#947.

\section{DEPARTMENT OF MATHEMATICS, STANFORD UNIVERSITY, STANFORD, CALIFORNIA 94305}

Current address: Department of Mathematics, Pennsylvania State University, University Park, Pennsylvania 16802 